TRANSACTIONS OF THE

AMERICAN MATHEMATICAL SOCIETY

Volume 357, Number 6, Pages 2253-2289

S 0002-9947(04)03566-4

Article electronically published on May 10, 2004

\title{
HOMOLOGICAL ALGEBRA FOR THE REPRESENTATION GREEN FUNCTOR FOR ABELIAN GROUPS
}

\author{
JOANA VENTURA
}

\begin{abstract}
In this paper we compute some derived functors Ext of the internal homomorphism functor in the category of modules over the representation Green functor. This internal homomorphism functor is the left adjoint of the box product.

When the group is a cyclic 2-group, we construct a projective resolution of the module fixed point functor, and that allows a direct computation of the graded Green functor Ext.

When the group is $G=\mathbb{Z} / 2 \times \mathbb{Z} / 2$, we can still build a projective resolution, but we do not have explicit formulas for the differentials. The resolution is built from long exact sequences of projective modules over the representation functor for the subgroups of $G$ by using exact functors between these categories of modules. This induces a filtration which gives a spectral sequence which converges to the desired Ext functors.
\end{abstract}

\section{INTRODUCTION}

Homotopy groups are the object of study in homotopy theory and stable homotopy theory. In the equivariant analogue of homotopy theory, we need to consider not just homotopy groups but invariants in a larger abelian category, which is called the category of Mackey functors. In the category $\operatorname{Mack}(G)$ of Mackey functors for a finite group $G$ there is a product, the box product $\square$ 3, 4, that has similar properties to the tensor product in $A b$. The objects $R$ with a ring structure, i.e. a product $R \square R \longrightarrow R$ and a unit satisfying some properties, are called Green functors. There are also notions of modules over a Green functor, bimodules, etc. The box product has a right adjoint $\mathcal{H}$. Given two Mackey functors $M$ and $N$, $\mathcal{H}(M, N)$ is the Mackey functor of internal homomorphisms from $M$ to $N$. We can also talk about the box product over a Green functor, $\square_{R}$, and the $R$-module internal homomorphism functor, $\mathcal{H}_{R}$, and they are adjoint. Tor, Ext, $\operatorname{Tor}^{R}$ and $\operatorname{Ext}_{R}$ are the derived functors of $\square, \mathcal{H}, \square_{R}$ and $\mathcal{H}_{R}$. They always exist because the categories of Mackey functors and of $R$-modules have enough projectives and injectives [4]. Roughly, there are the following analogies between Mackey functors

Received by the editors August 22, 2003.

2000 Mathematics Subject Classification. Primary 55P91, 18G10.

The author was partially supported by FCT grant Praxis XXI/BD/11357/97 and a one year research grant from Calouste Gulbenkian Foundation. 
and abelian groups:

$$
\begin{aligned}
\operatorname{Mack}(G) & \longleftrightarrow A b, \\
\text { Green functors } & \longleftrightarrow \text { rings with unit, } \\
\text { modules over a Green functor } R & \longleftrightarrow \text { modules over a ring } R, \\
\square, \mathcal{H}, \square_{R}, \mathcal{H}_{R} & \longleftrightarrow \otimes, \operatorname{Hom}, \otimes_{R}, \operatorname{Hom}_{R-\text { mod }}, \\
\text { Tor, Ext, } \operatorname{Tor}^{R}, \operatorname{Ext}_{R} & \longleftrightarrow \text { Tor, Ext, } \text { Tor }^{R}, \operatorname{Ext}_{R} .
\end{aligned}
$$

Functors Tor and Ext taken over the representation ring (when regarded as a Green functor) occur in Künneth and other spectral sequences for equivariant $K$ theory, when regarded as a Mackey functor. Also Tor and Ext over the category of Mackey functors arise in certain spectral sequences studied by Gunnar Carlsson for computing derived completions of representation rings. The computations made here were intended as a first step towards investigating those which would be needed to apply such spectral sequences.

We compute the Green functors $\operatorname{Ext}_{R}(F, F)=\bigoplus \operatorname{Ext}_{R}^{n}(F, F)$ when the group $G$ is $\mathbb{Z} / 2^{n}$ and $\mathbb{Z} / 2 \times \mathbb{Z} / 2$ (and when the ring of coefficients is $\mathbb{F}_{2}$ ), where $R$ is the representation Green functor (Example 1.5) and $F$ is the fixed point functor for the one-dimensional $\mathbb{F}_{2}$-vector space with trivial $G$-action (Example 1.6). These Ext functors at the $G$-set $G / e$ are always trivial.

Theorem A. Let $G=\mathbb{Z} / 2^{n}$. Then

$$
\operatorname{Ext}_{R}(F, F)(G / H)=\mathbb{F}_{2}\left[x_{4}\right] \otimes E\left(y_{3}\right)
$$

for all subgroups $H \neq e$, where $y_{3}$ has degree 3 and $x_{4}$ has degree 4 .

Theorem B. Let $G=\mathbb{Z} / 2 \times \mathbb{Z} / 2$. Then

$$
\begin{aligned}
& \operatorname{Ext}_{R}(F, F)(G / G)=\mathbb{F}_{2}\left[y_{3}, x_{4}\right] \otimes E\left(y_{5}\right), \\
& \operatorname{Ext}_{R}(F, F)(G / H)=\mathbb{F}_{2}\left[x_{4}\right] \otimes E\left(y_{3}\right) \text { for all } H \subseteq G \text { of index } 2,
\end{aligned}
$$

where $y_{3}$ has degree $3, x_{4}$ has degree 4 and $y_{5}$ has degree 5 . The restriction morphism from $\operatorname{Ext}_{R}(F, F)(G / G)$ to $\operatorname{Ext}_{R}(F, F)(G / H)$ is the ring map that sends $y_{3}$ and $x_{4}$ to the elements with the same name and sends $y_{5}$ to zero. Its transfer is zero.

The middle level (3) in Theorem B is isomorphic to the top level (1) in Theorem A when $n=1$. This is a general fact. The functor $\operatorname{Ext}_{R}(F, F)$ for a certain group $G$ at $G / H$ is the top level of the "same" functor for the group $H$ (Corollary 4.9).

In both cases, the classes $x_{4}$ come from a symmetry of a projective $R$-resolution of $F$. The classes $y_{i}$ seem to be a consequence of using $\mathbb{F}_{2}$ as the coefficient ring. Actually, when $G=\mathbb{Z} / 2 \times \mathbb{Z} / 2$, there is a class $x_{6}$ of degree 6 also coming from another symmetry, but $y_{3}^{2}=x_{6}$.

The obvious generalization of (2) for the groups $G=(\mathbb{Z} / 2)^{n}$ is

$$
\operatorname{Ext}_{R}(F, F)(G / G)=\mathbb{F}_{2}\left[y_{3}, x_{4}, y_{5}, x_{6}, \ldots, y_{2 n+1}, x_{2 n+2}\right] / J,
$$

where $J$ is the ideal generated by $y_{i}^{2}=x_{2 i}$ for $i \leq n+1$ and $y_{i}^{2}=0$ for $i>n+1$, with surjective restriction maps and zero transfers between two different orbits. Unfortunately this fails for $n=3$ and therefore for all larger $n$. When $n=3$, there are classes corresponding to $y_{3}$ and $x_{4}$, there is at least one class of degree 6 mapping to $x_{6}$ of (2) under the restriction map, and there is only one class of degree 5 , but it does not map to $y_{5}$ of (2). The computations for higher $n$ are much more difficult than for $n=2$; even considering only low degrees, it seems that there 
is no simple way of describing $\operatorname{Ext}_{R}(F, F)(G / G)$ since there is no obvious relation with $\operatorname{Ext}_{R}(F, F)(G / H)$ for other subgroups $H \subseteq G$.

This paper is the work done in my Ph.D. thesis and I thank Gunnar Carlsson for being a very patient advisor. I also thank the referee for suggesting a different construction in Section 4.2 .

\section{Part I. Background and notation}

\section{MACKey FUnCTORS}

There are several equivalent ways of defining a Mackey functor for a finite group [4, 7, 1. To fix notation and because that is what we will need in the rest of the paper, we will give two of these definitions. One is more abstract and is good to derive general properties, the other provides a better setting for the computations done in the second part.

Let $G$ be a finite group. Consider the category $\mathcal{B}^{+}(G)$ whose objects are finite $G$-sets and whose morphisms from $X$ to $Y$ are equivalence classes of diagrams

$$
X \stackrel{f}{\longleftarrow} V \stackrel{g}{\longrightarrow} Y,
$$

where $X, Y$ and $V$ are $G$-sets, $f$ and $g$ are $G$-maps. Two diagrams

$$
X \stackrel{f_{1}}{\longleftarrow} V_{1} \stackrel{g_{1}}{\longrightarrow} Y \quad \text { and } \quad X \stackrel{f_{2}}{\longleftarrow} V_{2} \stackrel{g_{2}}{\longrightarrow} Y
$$

are equivalent if there is an isomorphism $h: V_{1} \rightarrow V_{2}$ of $G$-sets such that the following diagram commutes:

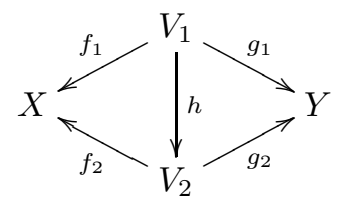

The composition of the morphisms represented by $X \stackrel{f_{1}}{\longleftarrow} V_{1} \stackrel{g_{1}}{\longrightarrow} Y$ and $Y \stackrel{f_{2}}{\longleftarrow}$ $V_{2} \stackrel{g_{2}}{\longrightarrow} Z$ is obtained from the pullback

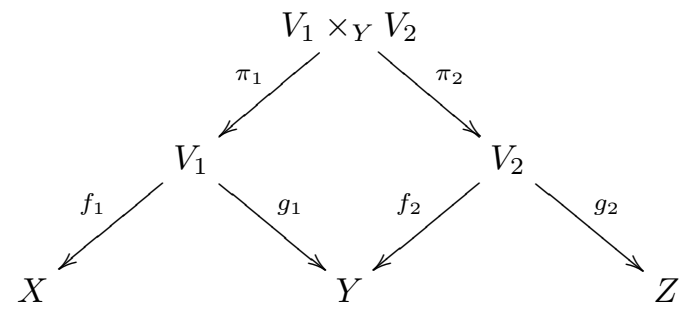

This gives a diagram $X \stackrel{f_{1} \pi_{1}}{\stackrel{n^{\prime}}{\leftrightarrows}} V_{1} \times_{Y} V_{2} \stackrel{g_{2} \pi_{2}}{\longrightarrow} Z$ that represents a morphism from $X$ to $Z$.

A $G$-map $f: X \longrightarrow Y$ induces two morphisms in $\mathcal{B}^{+}(G)$, one from $X$ to $Y$ represented by $X \stackrel{i d}{\longleftarrow} X \stackrel{f}{\longrightarrow} Y$ and the other from $Y$ to $X$ represented by $Y \stackrel{f}{\longleftarrow}$ $X \stackrel{i d}{\longrightarrow} X$. Denote the first by $f$ and the second by $\widehat{f}$. We will call $\widehat{f}$ the transfer map associated to $f$. So, any morphism in $\mathcal{B}^{+}(G)$ is the composition of morphisms of $G$-maps and transfer maps, more precisely, (1.1) is $g \widehat{f}$. 
The sets $\operatorname{Hom}_{\mathcal{B}^{+}(G)}(X, Y)$ are monoids under the disjoint union of the middle sets

$$
\left(X \longleftarrow V_{1} \longrightarrow Y\right)+\left(X \longleftarrow V_{2} \longrightarrow Y\right)=X \longleftarrow V_{1} \amalg V_{2} \longrightarrow Y
$$

and the zero corresponds to the diagram $X \longleftarrow \emptyset \longrightarrow Y$ with the empty set in the middle. Define a new category $\mathcal{B}(G)$, which is called the Burnside category, with the same objects as $\mathcal{B}^{+}(G)$ and where the group of morphisms $\operatorname{Hom}_{\mathcal{B}(G)}(X, Y)$ is the group completion of $\operatorname{Hom}_{\mathcal{B}^{+}(G)}(X, Y)$. The disjoint union of $G$-sets is a product and a coproduct, hence $\mathcal{B}(G)$ is an additive category. We can extend the coefficients to any commutative $\operatorname{ring} \mathcal{Z}$ with unit and still get an additive category. Denote it by $\mathcal{B}_{\mathcal{Z}}(G)$ or $\mathcal{B}(G)$ or just $\mathcal{B}$.

There is an obvious isomorphism $\mathcal{B} \longrightarrow \mathcal{B}^{o p}$ that sends the morphism represented by (1.1) to the morphism represented by $Y \stackrel{g}{\longleftarrow} V \stackrel{f}{\longrightarrow} X$.

Definition 1.1. A Mackey functor for the group $G$ with coefficients in $\mathcal{Z}$ is an additive contravariant functor from $\mathcal{B}(G)$ to the category of $\mathcal{Z}$-modules.

A morphism between Mackey functors is just a natural transformation. Denote by $\operatorname{Mack}(G)$ the category of Mackey functors for the group $G$. It is an abelian category. Kernels, cokernels and images of maps between Mackey functors, i.e. natural transformations, are defined objectwise.

Definition 1.2. For any Mackey functor $M$ and any finite $G$-set $X$, define a new Mackey functor $M_{X}$ as follows: $M_{X}(Y)=M(Y \times X)$ for all $G$-sets $Y$ and $M_{X}(\alpha)=M\left(\alpha \times \operatorname{Id}_{X}\right)$ for all morphisms $\alpha$ in $\mathcal{B}(G)$.

Since any $G$-set is a disjoint union of transitive sets and any morphism in $\mathcal{B}$ is a linear combination of compositions of morphisms induced by $G$-maps, to define a Mackey functor is enough to define it on transitive sets and on the morphisms induced by $G$-maps. For any $K \subseteq H \subseteq G$, let $\pi_{K}^{H}: G / K \longrightarrow G / H$ be the quotient map and let $\gamma_{x, H}: G / H \longrightarrow G /{ }^{x} H$ be the map $H \mapsto H x^{-1}=x^{-1}\left({ }^{x} H\right)$. Any $G$ map $f: G / K \longrightarrow G / H$ can be written as $\pi_{K^{x}}^{H} \gamma_{x^{-1}, K}$, where $K^{x} \subseteq H$ (or $K \subseteq{ }^{x} H$ ). So, a Mackey functor $M$ is completely determined by $M(G / H), M\left(\pi_{K}^{H}\right), M\left(\widehat{\pi}_{K}^{H}\right)$ and $M\left(\gamma_{x, H}\right)$ for all subgroups $K \subseteq H \subseteq G$ and $x \in G$. Notice that $\widehat{\gamma}_{x, H}$ is $\gamma_{x^{-1}, H}$.

Example 1.3. Let $A[H]$ be the Burnside ring of the group $H$. The Burnside functor $A$ is defined by $A(G / H)=A[H]$ for every subgroup $H \subseteq G$ and

- $A\left(\widehat{\pi}_{K}^{H}\right): A[K] \longrightarrow A[H]$ is $X \mapsto H \times_{K} X=H \times X / \sim$, where $\sim$ is the equivalence relation $(h k, x) \sim(h, k x), k \in K$,

- $A\left(\pi_{K}^{H}\right): A[H] \longrightarrow A[K]$ is restriction of the $H$-action,

- $A\left(\widehat{\gamma}_{x, H}\right): A[H] \longrightarrow A\left[{ }^{x} H\right]$ is defined on the transitive $H$-sets by $H / L \mapsto$ ${ }^{x} H /{ }^{x} L$ for all $L \subseteq H$ up to conjugacy in $H$.

The Burnside functor is also the representable functor $\operatorname{Hom}_{\mathcal{B}(G)}(\cdot, G / G)$.

Example 1.4. The representable functor $\operatorname{Hom}_{\mathcal{B}(G)}(\cdot, X)$ is $A_{X}$.

Example 1.5. Given a group $H$, let $R[H]$ be the representation ring over $\mathbb{C}$ of $H$. The representation functor $R$ is defined by $R(G / H)=R[H]$ for every subgroup $H \subseteq$ $G$. The morphism $R\left(\pi_{K}^{H}\right): R[H] \longrightarrow R[K], K \subseteq H$ is restriction of representations and $R\left(\widehat{\pi}_{K}^{H}\right): R[K] \longrightarrow R[H]$ is induction of representations. The $G$-map $\gamma_{x, H}$ induces the morphism $R\left(\widehat{\gamma}_{x, H}\right): R[H] \longrightarrow R\left[{ }^{x} H\right]$ that sends an $H$-vector space $V$ to the same vector space with ${ }^{x} H$-action given by $g \cdot v=x^{-1} g x v$, for all $g \in{ }^{x} H$ and $v \in V$. 
As suggested by the previous example, for any $G$-map $f$ and any Mackey functor $M, M(f)$ is called a restriction map and $M(\widehat{f})$ is called an induction map or transfer. A map $M\left(\gamma_{x, H}\right)$ is called conjugation.

Example 1.6. Let $M$ be a $\mathcal{Z}$-module with a $G$-action. The fixed point functor $F P_{M}$ is defined by $F P_{M}(G / H)=M^{H}=\{m \in M: h m=m \forall h \in H\}$, $F P_{M}\left(\pi_{K}^{H}\right)$ is the inclusion $M^{H} \subseteq M^{K}, F P_{M}\left(\widehat{\pi}_{K}^{H}\right)$ is the map $m \mapsto \sum_{h \in H / K} h m$, and $F P_{M}\left(\widehat{\gamma}_{x, H}\right): M^{H} \longrightarrow M^{x} H$ is $m \mapsto x m$.

As a particular case, consider $M=\mathcal{Z}$, the free rank $1 \mathcal{Z}$-module with trivial action. Then $F P_{\mathcal{Z}}(G / H)=\mathcal{Z}$ for all $H \subseteq G, F P_{\mathcal{Z}}(f)=1$ and $F P_{\mathcal{Z}}(\widehat{f})=[H: K]$ for all $G$-maps $G / K \stackrel{f}{\longrightarrow} G / H$.

It is well known that a small abelian category is equivalent to a category of modules over a certain algebra. For $\operatorname{Mack}(G)$ that algebra over $\mathcal{Z}$, denoted by $\mu(G)$ or $\mu$, is called Mackey algebra and is defined by

$$
\mu(G)=\bigoplus_{H, K \subseteq G} \operatorname{Hom}_{\mathcal{B}(G)}(G / K, G / H)
$$

with the multiplication defined by composition of morphisms in $\mathcal{B}(G)$ or zero if two morphisms cannot be composed [7].

It can also be defined as the algebra generated by $t_{K}^{H}, r_{K}^{H}$ and $c_{x, H}$, for $K \subseteq$ $H \subseteq G$ and $x \in G$ with the following relations:

$$
\begin{gathered}
t_{K}^{H} t_{L}^{K}=t_{L}^{H}, L \subseteq K \subseteq H, \\
r_{L}^{K} r_{K}^{H}=r_{L}^{H}, L \subseteq K \subseteq H, \\
c_{y,{ }^{x} H} c_{x, H}=c_{x y, H}, \\
t_{H}^{H}=r_{H}^{H}=c_{h, H}, h \in H, \\
c_{x, H} t_{K}^{H}=t_{x K}^{x H} c_{x, K}, \\
c_{x, K} r_{K}^{H}=r_{x}^{{ }^{x} H} c_{x, H}, \\
\sum_{K}^{H} t_{L}^{H}=t_{K \cap{ }^{x} L}^{K} c_{x, K} \cap L^{r} r_{K^{x} \cap L}^{L}, \\
\sum_{x \in K \backslash H / L} t_{H}^{H}=\sum_{H \subseteq G} r_{H}^{H}=1, \\
H \subseteq G \\
t_{H}^{H} t_{K}^{K}=0, H \neq K .
\end{gathered}
$$

The two definitions are equivalent [7], and an isomorphism is defined by sending $\widehat{\pi}_{K}^{H} \mapsto t_{K}^{H}, \pi_{K}^{H} \mapsto r_{K}^{H}$ and $\widehat{\gamma}_{x, H} \mapsto c_{x, H}$.

The identity morphism in $\operatorname{Hom}_{\mathcal{B}(G)}(G / H, G / H)$ corresponds to $t_{H}^{H}$. To simplify notation, let $1_{H}=t_{H}^{H}$. These elements are idempotents, hence the modules $\mu 1_{H}$ are projective.

As a $\mathcal{Z}$-module, $\mu(G)$ is free and the elements $t_{x_{L}}^{K} c_{x, L} r_{L}^{H}$, with $x \in K \backslash G / H$ and $L \subseteq H \cap K^{x}$ chosen up to $H \cap K^{x}$-conjugacy, form a basis (see 7]).

Theorem $1.7([7])$. The category of Mackey functors and the category of left $\mu$ modules are equivalent.

The equivalence $\operatorname{Mack}(G) \longrightarrow \mu$-mod sends a Mackey functor $M$ to $\widetilde{M}=$ $\bigoplus M(G / H)$, where the direct sum is over all subgroups $H \subseteq G$. This is a left 
$\mu$-module with $r_{K}^{H}$ acting as the restriction $M\left(\pi_{K}^{H}\right), t_{K}^{H}$ as the induction $M\left(\widehat{\pi}_{K}^{H}\right)$ and $c_{x, H}$ as the conjugation $M\left(\widehat{\gamma}_{x, H}\right)$. Conversely, the equivalence $\mu$-mod $\longrightarrow \operatorname{Mack}(G)$ sends a $\mu$-module $N$ to the Mackey functor $\bar{N}$ defined by $\bar{N}(G / H)=t_{H}^{H} N$ and the images of the morphisms in $\mathcal{B}(G)$ by the action of the corresponding elements in $\mu$. Using the first definition of $\mu$ and the fact that $A_{G / H}$ is the representable functor $\operatorname{Hom}_{\mathcal{B}(G)}(\cdot, G / H)$, we can see that

$$
\mu(G)=\bigoplus_{H, K \subseteq G} A_{G / H}(G / K)=\bigoplus_{H, K \subseteq G} A(G / K \times G / H)=A\left(\Omega^{2}\right),
$$

where $\Omega=\coprod_{H \subseteq G} G / H$. This also shows that the $\mu$-module corresponding to the Mackey functor $A_{G / H}$ is $\mu 1_{H}$.

Definition 1.8 (3]). Let $M$ and $N$ be Mackey functors, and let $Z$ be a finite $G$-set. The box product of $M$ and $N$, denoted $M \square N$, is the Mackey functor defined at $Z$ as the double coend

$$
M \square N(Z)=\iint^{X, Y} M(X) \otimes N(Y) \otimes \operatorname{Hom}_{\mathcal{B}}(Z, X \times Y)
$$

over the category $\mathcal{B}$. Naturality in $Z$ defines $M \square N$ on morphisms in $\mathcal{B}$.

The box product is commutative and associative, i.e., $M \square N \simeq N \square M$ and $(M \square N) \square P \simeq M \square(N \square P)$, and it has an adjoint functor which is the internal homomorphism functor $\mathcal{H}$.

Definition 1.9. Given Mackey functors $M$ and $N$, let $\mathcal{H}(M, N)$ be the Mackey functor defined by $\mathcal{H}(M, N)(X)=\operatorname{Hom}_{M a c k(G)}\left(M, N_{X}\right)$ on every $G$-set $X$.

Proposition 1.10 ([1]). $\mathcal{H}(M \square N, P) \simeq \mathcal{H}(N, \mathcal{H}(M, P))$, in particular,

$$
\operatorname{Hom}_{M a c k(G)}(M \square N, P) \simeq \operatorname{Hom}_{M a c k(G)}(N, \mathcal{H}(M, P)),
$$

i.e. $\square$ is left adjoint to $\mathcal{H}$.

As a corollary, we get that $\square$ is right exact and $\mathcal{H}(\cdot, P)$ is left exact.

Proposition 1.11 ([1, 4]). For any Mackey functor $M$ and any finite $G$-sets $X$ and $Y$, we have $A_{X} \square M \simeq M \square A_{X} \simeq M_{X}$, in particular $A_{X} \square A_{Y} \simeq A_{X \times Y}$, and $\mathcal{H}\left(A_{X}, M\right) \simeq M_{X}$.

The last isomorphism in Proposition 1.11 can be seen as a generalization of the isomorphism

$$
\phi: \operatorname{Hom}_{M a c k(G)}\left(A_{X}, M\right) \stackrel{\simeq}{\longrightarrow} M(X)
$$

which is the Yoneda Lemma: given a map $F: A_{X} \longrightarrow M$, then $\phi(F)$ is the image of $\operatorname{Id}_{X} \in A_{X}(X)=\operatorname{Hom}_{\mathcal{B}}(X, X)$ under $F(X): A_{X}(X) \longrightarrow M(X)$. Conversely, given $x \in M(X)$, the map $\phi^{-1}(x): A_{X} \longrightarrow M$ is defined at the object $Y$ by

$$
A_{X}(Y)=\operatorname{Hom}_{\mathcal{B}}(Y, X) \ni f \longmapsto M(f)(x) \in M(Y) .
$$

$\operatorname{Mack}(G)$ with the box product is a closed symmetric monoidal category.

Here is another very useful property of $\square$ and $\mathcal{H}$.

Proposition $1.12([1])$. There is an associative pairing

$$
\text { ô : } \mathcal{H}(N, P) \square \mathcal{H}(M, N) \longrightarrow \mathcal{H}(M, P)
$$

for any Mackey functors $M, N$ and $P$. 


\section{Green FUnCtors AND MODUles}

In this section we give the definitions and some properties of Green functors and modules over Green functors. Most properties are generalizations of some corresponding property for Mackey functors. In fact, after giving the main definitions, we could go to the previous section and replace the Burnside functor $A$ by a commutative Green functor $R$ to obtain the rest of this section. The main references are 4, [7] and [1].

Definition 2.1. A Green functor $R$ is a Mackey functor with two maps of Mackey functors: $A \stackrel{1_{R}}{\longrightarrow} R$ and a multiplication $R \square R \stackrel{m}{\longrightarrow} R$, where $A$ is the Burnside functor, making the following diagrams commute:
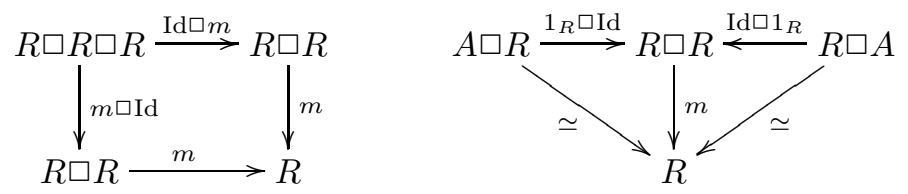

The first diagram says that the multiplication is associative, the second diagram says that $1_{R}$ is a left and a right unit in $R$. A map between Green functors is a map of Mackey functors making the obvious diagrams commute.

Definition 2.2. A Green functor $R$ is commutative if the diagram

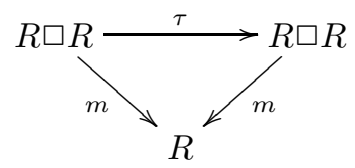

commutes, where $\tau$ permutes the coordinates and $m$ is the multiplication in $R$.

Definition 2.3. A (left) module over the Green functor $R$ is a Mackey functor $M$ with a map of Mackey functors $R \square M \stackrel{a}{\longrightarrow} M$ such that
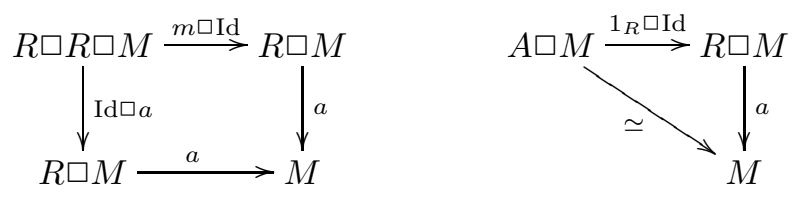

commute, where $m$ and $1_{R}$ are the multiplication and unit in $R$.

A map between two $R$-modules is a map of Mackey functors preserving the action of $R$. Denote by $R$-mod the category of (left) modules over the Green functor $R$. Similarly we can define right modules over $R$ and also bimodules.

The canonical isomorphism $A \square A \simeq A$ and the identity map $\operatorname{Id}_{A}$ give $A$ the structure of a Green functor. Any Mackey functor $M$ can be viewed as an $A$ module with the $A$-action given by the isomorphism $A \square M \simeq M$.

Green functors and modules over Green functors can also be defined as follows:

Definition 2.4. A Mackey functor $R$ is a Green functor if $R(X)$ is a $\mathcal{Z}$-algebra for any $G$-set $X$, the maps $R(f)$ are ring homomorphisms preserving the unit, and

$$
a R(\widehat{f})(b)=R(\widehat{f})(R(f)(a) b), \quad R(\widehat{f})(b) a=R(\widehat{f})(b R(f)(a))
$$

for any $G$-map $f: X \longrightarrow Y$ and $a \in R(X), b \in R(Y)$. 
Definition 2.5. Let $R$ and $S$ be Green functors. A morphism of Mackey functors $f: R \longrightarrow S$ is a morphism of Green functors if each $f(X)$ is a morphism of rings.

Definition 2.6. A Mackey functor $M$ is a (left) module over the Green functor $R$ if $M(X)$ is a (left) module over $R(X)$, and if $f: X \longrightarrow Y$ is a $G$-map, then

$$
\begin{aligned}
M(f)(a m) & =R(f)(a) M(f)(m) & & \forall a \in R(Y), m \in M(Y), \\
a M(\widehat{f})(m) & =M(\widehat{f})(R(f)(a) m) & & \forall a \in R(Y), m \in M(X), \\
R(\widehat{f})(a) m & =M(\widehat{f})(a M(f)(m)) & & \forall a \in R(X), m \in M(Y) .
\end{aligned}
$$

Definition 2.7. Let $M$ and $N$ be modules over the Green functor $R$. The morphism of Mackey functors $f: M \longrightarrow N$ is a morphism of $R$-modules if each $f(G / H)$ is a morphism of $R(G / H)$-modules.

These definitions are equivalent to the first ones ([1], [4]).

Example 2.8. With the second set of definitions, it is easy to check that the representation functor $R$ defined in Example 1.5 is a Green functor which is commutative because the ring $R(X)$ is commutative for all finite $G$-sets $X$. Also, the fixed point functor $F P_{\mathcal{Z}}$ (see Example 1.6) is an $R$-module ( $R$ with coefficients in $\mathcal{Z}$ ) with the $R$-action induced by the map $\epsilon: R \longrightarrow F P_{\mathcal{Z}}$ which is defined at each transitive $G$-set $G / H$ as the usual augmentation map $\epsilon(G / H): R[H] \longrightarrow \mathcal{Z}$ (tensored with the ring $\mathcal{Z}$ ), i.e. a representation $\sigma$ in $R(G / H)=R[H]$ acts on the module $F_{\mathcal{Z}}(G / H)=\mathcal{Z}$ by multiplication by $\operatorname{dim}(\sigma)$.

Fix a Green functor $R$. Other examples of Green functors are $R_{X}$, with $X$ any $G$-set. These are also $R$-modules. If $M$ is an $R$-module, then $M_{X}$ is a module over $R$ and over $R_{X}$.

Another important example of a Green functor is $\mathcal{H}(M, M)$, where $M$ is any Mackey functor. The product is given by the map of Proposition 1.12 and the unit $A \longrightarrow \mathcal{H}(M, M)$ is the map that sends $G / G \in A[G]$ to the identity map $\operatorname{Id}_{M} \in \operatorname{Hom}_{M a c k(G)}(M, M)=\mathcal{H}(M, M)(G / G)$.

For any Green functor $R$, we can define a category $\mathcal{C}_{R}$ as follows: the objects are finite $G$-sets, the set of morphisms from $X$ to $Y$ is

$$
R(X \times Y) \simeq \operatorname{Hom}_{R-\bmod }\left(R_{X}, R_{Y}\right)
$$

and the composition of morphisms in $\mathcal{C}_{R}$ is a composition of $R$-module morphisms. $\mathcal{C}_{R}$ plays the same role for $R$-modules as the Burnside category $\mathcal{B}$ for Mackey functors, which are $A$-modules. In fact we have $\mathcal{C}_{A}=\mathcal{B}$.

As in the case of the Burnside category, when $R$ is commutative, we also have a natural isomorphism $\mathcal{C}_{R} \longrightarrow \mathcal{C}_{R}^{o p}$ since

$$
\operatorname{Hom}_{\mathcal{C}_{R}}(X, Y) \simeq R(X \times Y) \simeq R(Y \times X) \simeq \operatorname{Hom}_{\mathcal{C}_{R}}(Y, X) .
$$

We will call it the opposite map.

Proposition 2.9 ([1]). The category of $R$-modules is equivalent to the category of additive contravariant functors from $\mathcal{C}_{R}$ to $\mathcal{Z}$-mod.

The unit of the Green functor $R, 1_{R}: A \longrightarrow R$, gives a functor $\mathcal{B} \longrightarrow \mathcal{C}_{R}$ since both categories have the same objects and $\operatorname{Hom}_{\mathcal{B}}(X, Y)=A(X \times Y)$ and $\operatorname{Hom}_{\mathcal{C}_{R}}(X, Y)=R(X \times Y)$. So $\mathcal{C}_{R}$ contains the morphisms in $\mathcal{B}$ (there might be 
some identifications), which are generated by $G$-maps and transfers; composition of these morphisms in $\mathcal{C}_{R}$ is the same as in $\mathcal{B}$. The other morphisms in $\mathcal{C}_{R}$ encode the action of the ring $R(X)$ on the $R(X)$-module $M(X)$, for any $R$-module $M$.

Notice that the representable functors $\operatorname{Hom}_{\mathcal{C}_{R}}(\cdot, X)$ are the $R$-modules $R_{X}$, by definition of the category $\mathcal{C}_{R}$. If $R$ is the Burnside functor $A$, we recover Example 1.4. For $R=A$, Proposition 2.9 only says that the category of $A$-modules is equivalent to the category of Mackey functors.

Now we can define an algebra in the same way the Mackey algebra was defined:

$$
\rho=\bigoplus_{H, K \subseteq G} \operatorname{Hom}_{\mathcal{C}_{R}}(G / K, G / H) \simeq R\left(\Omega^{2}\right)
$$

where $\Omega$ is the disjoint union of all transitive $G$-sets, and the product is given by composition of morphisms in $\mathcal{C}_{R}$ or zero if two morphisms cannot be composed.

Proposition 2.10 ([1]). The algebra $\rho$ is generated by $t_{K}^{H}, r_{K}^{H}, c_{x, H}, \lambda_{H, a}$ for $K \subseteq H \subseteq G, x \in G, a \in R(G / K)$. The relations among $t_{K}^{H}, r_{K}^{H}$ and $c_{x, H}$ are the same as in the Mackey algebra, (1.2). The other relations are

$$
\begin{gathered}
\lambda_{K, a}+\lambda_{K, b}=\lambda_{K, a+b}, a, b \in R(G / K), \\
\lambda_{K, a} \lambda_{K, b}=\lambda_{K, a b}, a, b \in R(G / K), \\
\lambda_{K, z 1}=z t_{K}^{K}, z \in \mathcal{Z}, \text { where } 1 \text { is the unit of } R(G / K), \\
r_{K}^{H} \lambda_{H, a}=\lambda_{K, r_{K}^{H}(a)} r_{K}^{H}, a \in R(G / H), K \subseteq H \subseteq G, \\
\lambda_{H, a} t_{K}^{H}=t_{K}^{H} \lambda_{K, r_{K}^{H}(a)}, a \in R(G / H), K \subseteq H \subseteq G, \\
c_{x, H} \lambda_{H, a}=\lambda_{x, c_{x, H}(a)} c_{x, H}, a \in R(G / H), x \in G, \\
t_{K}^{H} \lambda_{K, a} r_{K}^{H}=\lambda_{H, t_{K}^{H}(a)}, a \in R(G / K), K \subseteq H \subseteq G .
\end{gathered}
$$

The opposite map $\mathcal{C}_{R} \longrightarrow \mathcal{C}_{R}^{o p}$ induces an anti-isomorphism of the algebra $\rho$ that sends an element $v$ to its transfer $\widehat{v}$. On the generators of $\rho$, it is given by

$$
\widehat{r}_{K}^{H}=t_{K}^{H}, \quad \widehat{t}_{K}^{H}=r_{K}^{H}, \quad \widehat{c}_{x, H}=c_{x^{-1},{ }^{x} H} \quad \text { and } \quad \widehat{\lambda}_{H, a}=\lambda_{H, a} .
$$

Proposition 2.11 ([1]). The category of left $R$-modules is equivalent to the category of left $\rho$-modules.

The isomorphism $\rho$-mod $\longrightarrow R$-mod is $V \mapsto M_{V}$, where $M_{V}(X)=R(X \times \Omega)$ $\otimes_{R\left(\Omega^{2}\right)} V$ for all $G$-sets $X$ and $\Omega=\bigsqcup_{H \subseteq G} G / H$. If $X=G / H$, then

$$
M_{V}(G / H)=R(G / H \times \Omega) \otimes_{R\left(\Omega^{2}\right)} V=\left(1_{H} \rho\right) \otimes_{\rho} V=1_{H} V
$$

because $R(G / H \times \Omega)=\operatorname{Hom}_{\mathcal{C}_{R}}(G / H, \Omega)=1_{H} \rho$. The isomorphism from $R$-mod to $\rho$-mod is $M \mapsto M(\Omega)$. If $M=R_{G / H}$, then

$$
R_{G / H}(\Omega)=R(\Omega \times G / H)=\operatorname{Hom}_{\mathcal{C}_{R}}(\Omega, G / H)=\rho 1_{H} .
$$

We now finish this section with the definitions of the box product over $R, \square_{R}$, and the internal homomorphism functor over $R, \mathcal{H}_{R}$, a subfunctor of $\mathcal{H}$, and a list of properties that $\square_{R}$ and $\mathcal{H}_{R}$ satisfy.

Definition 2.12. Let $M$ be a right $R$-module and $N$ a left $R$-module. The Mackey functor $M \square_{R} N$ is the coequalizer of $M \square R \square N \stackrel{a_{M} \square i d}{\longrightarrow} M \square N$ and $M \square R \square N \stackrel{i d \square a_{N}}{\longrightarrow}$ $M \square N$, where $a_{M}$ and $a_{N}$ are the actions of $R$ in $M$ and $N$. 
Definition 2.13. Let $M$ and $N$ be left $R$-modules. $\mathcal{H}_{R}(M, N)$ is the Mackey functor defined by $\mathcal{H}_{R}(M, N)(X)=\operatorname{Hom}_{R-\bmod }\left(M, N_{X}\right)$ for all $G$-sets $X$.

Proposition 2.14 ([1]). Let $M$ be a bimodule, and let $N$ and $P$ be left modules. Then

$$
\mathcal{H}_{R}\left(M \square_{R} N, P\right) \simeq \mathcal{H}_{R}\left(N, \mathcal{H}_{R}(M, P)\right) .
$$

In particular, $\operatorname{Hom}_{R-\bmod }\left(M \square_{R} N, P\right) \simeq \operatorname{Hom}_{R \text {-mod }}\left(N, \mathcal{H}_{R}(M, P)\right)$.

Corollary 2.15. $\mathcal{H}_{R}(\cdot, P)$ is left exact, and $\square_{R}$ is right exact.

For simplicity, from now on assume that $R$ is a commutative Green functor.

Proposition 2.16 ([4]). If $R$ is commutative and $M$ and $N$ are $R$-modules, then $M \square_{R} N$ and $\mathcal{H}_{R}(M, N)$ are $R$-modules, and $M \square_{R} N \simeq N \square_{R} M$.

Since the categories of left modules, right modules and bimodules over a commutative Green functor are all equivalent, Proposition 2.14 says that $\square_{R}$ and $\mathcal{H}_{R}$ are adjoint functors in the category $R$-mod if $R$ is commutative, i.e. $R$-mod is a closed symmetric monoidal category, just like $\operatorname{Mack}(G)$.

Proposition 2.17. $R_{X} \square_{R} M \simeq M \square_{R} R_{X} \simeq M_{X}$ and $\mathcal{H}_{R}\left(R_{X}, M\right) \simeq M_{X}$, for any $R$-module $M$.

This is the analogue of Proposition 1.11 and the second part is a generalization of

$$
\operatorname{Hom}_{R-\bmod }\left(R_{X}, M\right) \simeq M(X)
$$

which is again the Yoneda lemma as in (1.3).

Proposition 2.18 ([1]). If $M$ is an $R$-module, then $\mathcal{H}_{R}(M, M)$ is a Green functor with the product ô defined in Proposition 1.12.

Proposition 2.19. The product ô defined in Proposition 1.12 induces an associative pairing

$$
\mathcal{H}_{R}(N, P) \square \mathcal{H}_{R}(M, N) \longrightarrow \mathcal{H}_{R}(M, P) .
$$

\section{Homological Algebra}

Lewis in [4] shows that $\operatorname{Mack}(G)$ and the categories of left and right $R$-modules are abelian categories with enough injectives and projectives. The projectives in $\operatorname{Mack}(G)$ are generated, as modules, by the representable functors $A_{X}$. The projectives in $R$-mod are generated by $R_{X}$; they are representable in the category of functors from $\mathcal{C}_{R}$ to $\mathcal{Z}$-modules, which is equivalent to $R$-mod (Proposition 2.9). Let Tor, Ext, Tor ${ }^{R}$ and $\operatorname{Ext}_{R}$ be the derived functors for $\square, \mathcal{H}, \square_{R}$ and $\mathcal{H}_{R}$, respectively. By definition, $\operatorname{Ext}_{R}^{n}(M, N)$ and $\operatorname{Tor}_{n}^{R}(M, N)$ are just Mackey functors if $M$ and $N$ are $R$-modules. If $R$ is commutative, then $\mathcal{H}_{R}(M, N)$ and $M \square_{R} N$ are $R$ modules and that induces an $R$-module structure on $\operatorname{Ext}_{R}^{n}(M, N)$ and $\operatorname{Tor}_{n}^{R}(M, N)$. Moreover the graded Mackey functor $\operatorname{Ext}_{R}(M, M)=\bigoplus_{n>0} \operatorname{Ext}_{R}^{n}(M, M)$ is a Green functor with a Yoneda product induced by the pairing of Proposition2.19 on the $\mathcal{H}$ cochain complex, which is constructed in the same way the Hom cochain complex is for ordinary modules over a ring.

In the category of modules over an ordinary ring, we have the following standard results that we will need later. 
Proposition 3.1 (The short exact sequence trick). Given a short exact sequence of modules over a fixed ring

$$
0 \longrightarrow A \stackrel{i}{\longrightarrow} B \stackrel{j}{\longrightarrow} C \longrightarrow 0
$$

a projective resolution $\left(P_{*}, d_{*}\right)$ for $A$ and a projective resolution $\left(Q_{*}, \delta_{*}\right)$ for $C$, there is a projective resolution for $B$ of the form

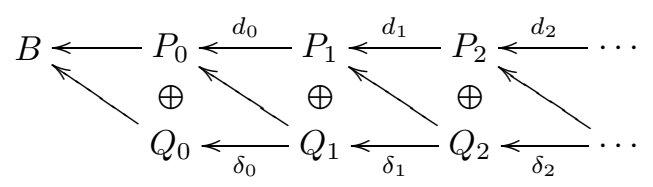

We are assuming the sign conventions in [9], namely if $(C, d)$ is a chain complex, then the $n^{\text {th }}$ translate of $C, C[n]$, is the complex defined by $C[n]_{i}=C_{i+n}$ with differentials $(-1)^{n} d$.

Lemma 3.2. Let $P_{*}$ be a projective resolution for a module $M$

$$
0 \longleftarrow M \stackrel{\epsilon}{\longleftarrow} P_{0} \stackrel{\partial_{0}}{\longleftarrow} P_{1} \stackrel{\partial_{1}}{\longleftarrow} P_{2} \longleftarrow \cdots
$$

and let $Q_{*}$ be a projective resolution for a module $N$

$$
0 \longleftarrow N \stackrel{\epsilon}{\longleftarrow} Q_{0} \stackrel{\partial_{0}}{\longleftarrow} Q_{1} \stackrel{\partial_{1}}{\longleftarrow} Q_{2} \longleftarrow \cdots .
$$

Let $f: P_{*} \longrightarrow Q[-n]_{*}$ be a chain map. Let $T_{*}$ be the chain complex with $T_{n}=N$ and $T_{i}=0$ if $i \neq n$. Let $\tilde{\epsilon}: Q[-n]_{*} \longrightarrow T_{*}$ be the chain map defined by $\tilde{\epsilon}_{n}=$ $(-1)^{n} \epsilon: Q_{0} \longrightarrow N$ and $\tilde{\epsilon}_{i}=0$ for $i \neq n$, where $\epsilon: Q_{0} \longrightarrow N$ is the augmentation map. Then $f$ is chain homotopic to zero if and only if $\tilde{\epsilon} f: P_{*} \longrightarrow T_{*}$ is chain homotopic to zero.

Proposition 3.3. $\operatorname{Ext}_{R}^{n}(M, N) \simeq\left[P_{*}, Q[-n]_{*}\right]$.

$\left[P_{*}, Q[-n]_{*}\right]$ denotes the group of chain homotopy equivalence classes of chain maps $P_{*} \longrightarrow Q[-n]_{*}$. The isomorphism sends a chain map $f: P_{*} \longrightarrow Q[-n]_{*}$ to the class represented by $(-1)^{n} \epsilon f$. Lemma 3.2 shows that this map is injective. See 9] for more details.

\section{Some exact Functors}

In this section we describe some exact functors defined on the category of modules over a Green functor. They are used in Part I to reduce the computation of the Green functor $\operatorname{Ext}_{R}(F, F)$ to the computation of the algebra $\operatorname{Ext}_{R}(F, F)(G / G)$, and also to build projective resolutions.

4.1. Induction and restriction. Fix a subgroup $H$ of $G$. Given a $G$-set $X$, let $\operatorname{Res}_{H}^{G} X$ denote the $H$-set obtained from $X$ by restricting the action. For any $H$-set $Y$, let $\operatorname{Ind}_{H}^{G} Y$ be the $G$-set $G \times_{H} Y$ defined in Example $1.3 \operatorname{Res}_{H}^{G}$ and $\operatorname{Ind}_{H}^{G}$ are the restriction map $A\left(\pi_{H}^{G}\right)$ and the induction map $A\left(\widehat{\pi}_{H}^{G}\right)$ in Example1.3 Now define the functors $\operatorname{Res}_{H}^{G}: \operatorname{Mack}(G) \longrightarrow \operatorname{Mack}(H)$ and $\operatorname{Ind}_{H}^{G}: \operatorname{Mack}(H) \longrightarrow \operatorname{Mack}(G)$ by

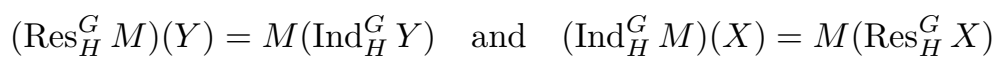

for every $H$-set $Y$ and $G$-set $X$.

Proposition 4.1 ([8]). $\operatorname{Res}_{H}^{G}: \operatorname{Mack}(G) \longrightarrow \operatorname{Mack}(H)$ is left and right adjoint of $\operatorname{Ind}_{H}^{G}: \operatorname{Mack}(H) \longrightarrow \operatorname{Mack}(G)$. 
Proposition $4.2([1]) \cdot \mathcal{H}(M, N)(G / H) \simeq \operatorname{Hom}_{M a c k(H)}\left(\operatorname{Res}_{H}^{G} M, \operatorname{Res}_{H}^{G} N\right)$.

The functors $\operatorname{Res}_{H}^{G}$ and $\operatorname{Ind}_{H}^{G}$ also have good properties when considering only Green functors or modules over a fixed Green functor. In [1 Bouc proves the following results.

Proposition 4.3. Let $R$ be a Green functor for the group $G$ and let $M$ be an $R$-module. Then $\operatorname{Res}_{H}^{G} R$ is a Green functor for $H$ and $\operatorname{Res}_{H}^{G} M$ is a module over $\operatorname{Res}_{H}^{G} R$. If $N$ is a $\operatorname{Res}_{H}^{G} R$-module, then $\operatorname{Ind}_{H}^{G} N$ is a module over $R$.

So we have well-defined functors

$$
\operatorname{Res}_{H}^{G}: R-\bmod \longrightarrow \operatorname{Res}_{H}^{G} R-\bmod
$$

and

$$
\operatorname{Ind}_{H}^{G}: \operatorname{Res}_{H}^{G} R \text {-mod } \longrightarrow R \text {-mod . }
$$

Proposition 4.4. The restriction functor $\operatorname{Res}_{H}^{G}$ is left and right adjoint to the induction $\operatorname{Ind}_{H}^{G}$.

Corollary 4.5. $\operatorname{Res}_{H}^{G}$ and $\operatorname{Ind}_{H}^{G}$ are exact.

Proposition 4.6. Let $M$ and $N$ be $R$-modules and $H \subseteq G$. Then

$$
\begin{aligned}
\mathcal{H}_{R}(M, N)(G / H) & \simeq \operatorname{Hom}_{\operatorname{Res}_{H}^{G} R-\bmod }\left(\operatorname{Res}_{H}^{G} M, \operatorname{Res}_{H}^{G} N\right) \\
& =\mathcal{H}_{\operatorname{Res}_{H}^{G} R}\left(\operatorname{Res}_{H}^{G} M, \operatorname{Res}_{H}^{G} N\right)(H / H) .
\end{aligned}
$$

An obvious and useful corollary is:

Corollary 4.7. Let $M, N$ be R-modules. Then

$$
\operatorname{Ext}_{R}^{n}(M, N)(G / H) \simeq \operatorname{Ext}_{\operatorname{Res}_{H}^{n} R-\bmod }\left(\operatorname{Res}_{H}^{G} M, \operatorname{Res}_{H}^{G} N\right)(H / H)
$$

for any subgroup $H \subseteq G$.

Now let $R^{G}$ and $R^{H}$ denote the representation functors for $G$ and $H$, respectively. In the same way, let $F^{G}$ and $F^{H}$ denote the fixed point functors for the groups $G$ and $H$.

Lemma 4.8. $\operatorname{Res}_{H}^{G} R^{G}=R^{H}$ and $\operatorname{Res}_{H}^{G} F^{G}=F^{H}$.

Proof. Trivial by the definitions of $R^{G}, R^{H}, F^{G}$ and $F^{H}$.

Corollary 4.9. $\operatorname{Ext}_{R^{G}}\left(F^{G}, F^{G}\right)(G / H) \simeq \operatorname{Ext}_{R^{H}}\left(F^{H}, F^{H}\right)(H / H)$.

Proof. Apply Corollary 4.7 with $R$ the representation functor for $G$ and $M=N=$ $F^{G}$.

Corollary 4.10. $\operatorname{Ext}_{R^{G}}^{n}\left(F^{G}, F^{G}\right)(G / e)=0$ for all $n>0$.

Proof. Since $\operatorname{Res}_{e}^{G} R^{G}=\mathcal{Z}$, the category of modules over the Green functor $\operatorname{Res}_{e}^{G} R^{G}$ is the category of $\mathcal{Z}$-modules. The module $\operatorname{Res}_{e}^{G} F^{G}=\mathcal{Z}$ is free, so

$$
\operatorname{Ext}_{R^{G}}^{n}\left(F^{G}, F^{G}\right)(G / e) \simeq \operatorname{Ext}_{\mathcal{Z} \text {-mod }}^{n}(\mathcal{Z}, \mathcal{Z})=0 .
$$


4.2. The upside down functor $\mathcal{U}$. In this subsection $G$ is a finite abelian group and $R$ is the representation Green functor for $G$ and with coefficients in any commutative ring $\mathcal{Z}$ with unit. The goal is to define an exact functor $\mathcal{U}: R$-mod $\longrightarrow R$-mod that will allow us to build a projective resolution in Sections 6 and 7 in half the steps we would have to do without it. The functor $\mathcal{U}$ is induced by an isomorphism $\iota$ of the algebra $\rho$ associated to the functor $R$.

Note. $R(X)$ denotes the value of the functor $R$ at the $G$-set $X$ and $R[H]$ denotes the representation algebra of the group $H$. So $R(G / H)$ is the value of the functor $R$ at $G / H$, i.e. $R(G / H)=R[H]$ and $G / H$ is just a $G$-set. The representation algebra $R[G / H]$ is not the same as $R(G / H)$, the first only makes sense if $H$ is normal in $G$ (which is always true for abelian groups), and the latter is always defined even if the group is not abelian.

For any finite abelian group $G$, we define its dual to be

$$
G^{*}=\operatorname{Hom}(G, \mathbb{Q} / \mathbb{Z}) .
$$

There is a pairing $G^{*} \times G \longrightarrow \mathbb{Q} / \mathbb{Z}$ defined by $(\sigma, g) \mapsto \sigma(g)$, i.e. it is the evaluation of a map $\sigma \in G^{*}$ at $g \in G$. Given $H \subseteq G$, let

$$
H^{\perp}=\left\{\sigma \in G^{*}: \sigma(h)=0 \text { for all } h \in H\right\} .
$$

$H^{\perp}$ is a subgroup of $G^{*}$. Since $G$ is abelian, $G$ is isomorphic to its dual. Fix an isomorphism $\varphi: G^{*} \longrightarrow G$ and let $H^{\prime}=\varphi\left(H^{\perp}\right)$. The functor $\mathcal{U}$ depends on this isomorphism $\varphi$.

Proposition 4.11. For any subgroups $K$ and $H$ of $G$, we have:

(i) $(K \cap H)^{\prime}=K^{\prime} H^{\prime}$.

(ii) $(K H)^{\prime}=K^{\prime} \cap H^{\prime}$.

(iii) If $K \subseteq H$ then $H^{\prime} \subseteq K^{\prime}$ and $[H: K]=\left[K^{\prime}: H^{\prime}\right]$.

Let $\rho$ be the algebra associated to $R$ defined in Section 2 . Its generators are $t_{K}^{H}, r_{K}^{H}, c_{x, H}$, where $x$ are representatives of the left cosets $G / H$, and $\lambda_{H, \sigma}$ where $\sigma \in R[H]$. It is enough to consider $\lambda_{H, \sigma}$ for irreducible characters. If $\sigma$ is not irreducible, then it is a linear combination of irreducible characters $\sigma=\sum z_{i} \sigma_{i}$ and, applying (2.1a) and (2.1c), $\lambda_{H, \sigma}=\sum z_{i} \lambda_{H, \sigma_{i}}$.

Extend the isomorphism $\varphi: G^{*} \longrightarrow G$ used to define $H^{\prime}$ to the group algebra $\mathcal{Z}\left[G^{*}\right]$. Since the group $G$ is abelian, we identify $\mathcal{Z}\left[G^{*}\right]$ with $R[G]$, so that

$$
r_{H}^{G}: \mathcal{Z}\left[G^{*}\right] \longrightarrow \mathcal{Z}\left[H^{*}\right] \quad \text { and } \quad t_{H}^{G}: \mathcal{Z}\left[H^{*}\right] \longrightarrow \mathcal{Z}\left[G^{*}\right]
$$

denote the usual restriction and induction maps between $R[G]$ and $R[H]$. For each $\sigma \in \mathcal{Z}\left[H^{*}\right]$ choose $\chi \in \mathcal{Z}\left[G^{*}\right]$ such that $r_{H}^{G}(\chi)=\sigma$. Such $\chi$ exists because $r_{H}^{G}$ is surjective $\left(G\right.$ is abelian). Let $\psi_{H}(\sigma)=p \varphi(\chi)$, where $p: G \longrightarrow G / H^{\prime}$ is the quotient map. If $r_{H}^{G}\left(\chi_{1}\right)=r_{H}^{G}\left(\chi_{2}\right)$ for two characteres $\chi_{1}, \chi_{2} \in G^{*}$, then $\chi_{1} \chi_{2}^{-1} \in H^{\perp}$ and so $p \varphi\left(\chi_{1} \chi_{2}^{-1}\right)=1$, i.e. $p \varphi\left(\chi_{1}\right)=p \varphi\left(\chi_{2}\right)$. So we get a well-defined map of algebras $\psi_{H}: \mathcal{Z}\left[H^{*}\right] \longrightarrow \mathcal{Z}\left[G / H^{\prime}\right]$, which is obviously an isomorphism. The map $\psi_{H}$ was defined so that, with $p^{-1}(x)=\sum_{p(y)=x} y$, the following diagrams are commutative:

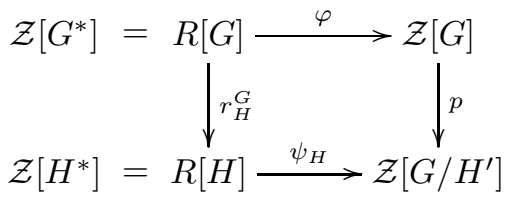

and

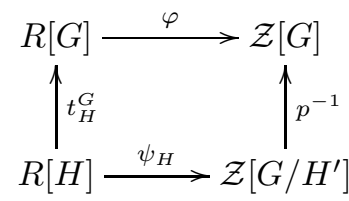


Now we will define the algebra homomorphism $\iota: \rho \longrightarrow \rho$.

Since $\rho \simeq R\left(\Omega^{2}\right)$, where $\Omega=\bigoplus_{H \subseteq G} G / H$, for every Green functor over any finite group $G$, there is a $\mathcal{Z}$-module isomorphism

$$
\rho \simeq \bigoplus_{H, K \subseteq G} \bigoplus_{x \in H \backslash G / K} R\left(G /\left(H \cap{ }^{x} K\right)\right),
$$

whose inverse is given by

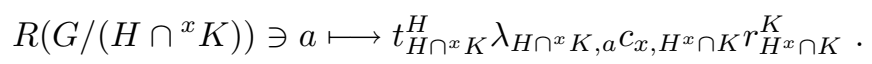

If $G$ is abelian, then

$$
\rho \simeq \bigoplus_{H, K \subseteq G} \mathcal{Z}[G / H K] R\left(G /\left(H \cap{ }^{x} K\right)\right) .
$$

If we also assume that $R$ is the representation functor, then

$$
R(G /(H \cap K))=R[H \cap K] \simeq \mathcal{Z}\left[(H \cap K)^{*}\right] \simeq \mathcal{Z}\left[G / H^{\prime} K^{\prime}\right],
$$

where the last isomorphism is the map $\psi_{H \cap K}$ (together with Proposition 4.11) defined above. Thus

$$
\rho \simeq \bigoplus_{H, K \subseteq G}\left(\mathcal{Z}[G / H K] \otimes \mathcal{Z}\left[G / H^{\prime} K^{\prime}\right]\right)
$$

If $H, K \subseteq G, u \in \mathcal{Z}[G / H K]$ and $u^{\prime} \in \mathcal{Z}\left[G / H^{\prime} K^{\prime}\right]$, denote by $\left[u \otimes u^{\prime}\right]_{H, K}$ the element $u \otimes u^{\prime}$ in the $(H, K)$-component of this decomposition.

Define the product in the right-hand side of (4.2) of the elements $\left[u \otimes u^{\prime}\right]_{H, K}$ and $\left[v \otimes v^{\prime}\right]_{L, M}$ by 0 if $K \neq L$, and by

$$
\gamma_{K, H, M} \sum_{\substack{x \in H K M / H M \\ x^{\prime} \in H^{\prime} K^{\prime} M^{\prime} / H^{\prime} M^{\prime}}}\left[x u v \otimes x^{\prime} u^{\prime} v^{\prime}\right]_{H, M}
$$

otherwise, where

$$
\gamma_{K, H, M}=[(K \cap H M):(K \cap H)(K \cap M)] .
$$

Lemma 4.12. The $\mathcal{Z}$-module isomorphim (4.2) is an algebra isomorphism.

Proof. To simplify notation, in this proof we will drop the index in the maps $\psi_{H}$, and denote by $\lambda_{a}$ and $c_{x}$ the elements $\lambda_{H, a}$ and $c_{x, H}$, respectively, where $a \in$ $R(G / H)$ and $x \in H$.

The inverse isomorphism given in (4.2) sends the element $\left[u \otimes u^{\prime}\right]_{H, K}$ to $\alpha:=$ $t_{H \cap K}^{H} \lambda_{\psi^{-1}\left(u^{\prime}\right)} c_{u} r_{K \cap H}^{K}$, and $\left[v \otimes v^{\prime}\right]_{L, M}$ to $\beta:=t_{L \cap M}^{L} \lambda_{\psi^{-1}\left(v^{\prime}\right)} c_{v} r_{L \cap M}^{M}$.

If $L \neq K$, then the product $\alpha \beta$ is 0 . If $L=K$, applying the relations (1.2) and (2.1) in the algebra $\rho$ to simplify the product $\alpha \beta$, we get

$$
\alpha \beta=\sum_{x \in K /(H \cap K)(K \cap M)} t_{H \cap K \cap M}^{H} \lambda_{\left.r_{H \cap K \cap K M} \cap \psi^{-1}\left(u^{\prime}\right)\right) r_{H \cap K \cap M}^{K \cap M}\left(\psi^{-1}\left(v^{\prime}\right)\right)} c_{x u v} r_{M \cap K \cap H}^{M} .
$$

If $x \in K \cap H M$, then $t_{H \cap K \cap M}^{H} c_{x u v} r_{H \cap K \cap M}^{M}=t_{H \cap K \cap M}^{H} c_{u v} r_{H \cap K \cap M}^{M}$. Also, since

$$
\begin{aligned}
& t_{H \cap K \cap M}^{H \cap M} \lambda_{r_{H \cap K \cap K}^{H \cap K}}\left(\psi^{-1}\left(u^{\prime}\right)\right) r_{H \cap K \cap M}^{K \cap M}\left(\psi^{-1}\left(v^{\prime}\right)\right) r_{H \cap K \cap M}^{H \cap M} \\
& =\lambda_{t H \cap K \cap M}\left(r_{H \cap K \cap K}^{H \cap K}\left(\psi^{-1}\left(u^{\prime}\right)\right) r_{H \cap K \cap M}^{K \cap M}\left(\psi^{-1}\left(v^{\prime}\right)\right)\right) \\
& =\sum_{x^{\prime} \in H^{\prime} K^{\prime} M^{\prime} / H^{\prime} M^{\prime}} \lambda_{H \cap M, \psi^{-1}\left(x^{\prime} u^{\prime} v^{\prime}\right)}
\end{aligned}
$$


(the first equality is relation (2.1g), and the second follows from the definition of the isomorphisms $\psi$ and the commutative diagrams (4.1)), the product $\alpha \beta$ simplifies to

$$
\alpha \beta=\gamma_{K, H, M} \sum_{\substack{x \in K /(K \cap H M) \\ x^{\prime} \in H^{\prime} K^{\prime} M^{\prime} / H^{\prime} M^{\prime}}} t_{H \cap M}^{H} \lambda_{\psi^{-1}\left(x^{\prime} u^{\prime} v^{\prime}\right)} c_{x u v} r_{H \cap M}^{M},
$$

which is the image of (4.3).

Let $\bar{\imath}$ be the map defined on the right-hand side of (4.2) that sends the element $\left[u \otimes u^{\prime}\right]_{H, K}$ to the element $\left[u^{\prime} \otimes u\right]_{H^{\prime}, K^{\prime}}$.

Lemma 4.13. The map $\bar{\iota}$ is an algebra homomorphism.

Proof. It is enough to show that $\gamma_{H, K, M}=\gamma_{H^{\prime}, K^{\prime}, M^{\prime}}$. By Proposition 4.11 we have

$$
\left(K^{\prime} \cap H^{\prime} M^{\prime}\right) /\left(\left(K^{\prime} \cap H^{\prime}\right)\left(K^{\prime} \cap M^{\prime}\right)\right)=(K(H \cap M))^{\prime} /(K H \cap K M)^{\prime}
$$

and so

$$
\begin{aligned}
\gamma_{H^{\prime}, K^{\prime}, M^{\prime}} & =\left[(K(H \cap M))^{\prime}:(K H \cap K M)^{\prime}\right] \\
& =[K H \cap K M: K(H \cap M)] .
\end{aligned}
$$

Using the group isomorphism

$$
(K \cap H M) /((K \cap H)(K \cap K)) \simeq(K H \cap K M) /(K(H \cap M)),
$$

we finally get $\gamma_{H, K, M}=\gamma_{H^{\prime}, K^{\prime}, M^{\prime}}$.

Denote by $\iota$ the conjugate of $\bar{\iota}$ by the algebra isomorphism (4.2). Since $\bar{\iota}$ is an algebra isomorphism, this map $\iota: \rho \longrightarrow \rho$ is also an algebra isomorphism.

Proposition 4.14. The map $\iota: \rho \longrightarrow \rho$ is an algebra isomorphism such that $\iota^{2}=i d$ and

$$
\begin{gathered}
\iota\left(t_{K}^{H}\right)=r_{H^{\prime}}^{K^{\prime}}, \quad \iota\left(r_{K}^{H}\right)=t_{H^{\prime}}^{K^{\prime}}, \\
\iota\left(c_{x, H}\right)=\lambda_{H^{\prime}, \psi_{H^{\prime}}^{-1}(x)} \quad \text { and } \quad \iota\left(\lambda_{H, \sigma}\right)=c_{\psi_{H}(\sigma), H^{\prime}}
\end{gathered}
$$

if $\sigma \in R[H]$ is irreducible.

Proof. Obviously $\iota^{2}=i d$ because $\bar{\iota}$ also satisfies this property.

The isomorphim (4.2) interchanges the following pairs of elements:

$$
\begin{aligned}
t_{K}^{H} & \longleftrightarrow[\otimes 1]_{H, K} \quad \text { if } K \subseteq H, \\
r_{K}^{H} & \longleftrightarrow[1 \otimes 1]_{K, H} \quad \text { if } K \subseteq H, \\
c_{x, H} & \longleftrightarrow[x \otimes 1]_{H, H}, \\
\lambda_{H, \sigma} & \longleftrightarrow\left[1 \otimes \psi_{H}(\sigma)\right]_{H, H} .
\end{aligned}
$$

Applying the map $\bar{\iota}$ to the elements on the right, we get respectively the following four elements:

$$
[1 \otimes 1]_{H^{\prime}, K^{\prime}}, \quad[1 \otimes 1]_{K^{\prime}, H^{\prime}}, \quad[1 \otimes x]_{H^{\prime}, H^{\prime}} \quad \text { and } \quad\left[\psi_{H}(\sigma) \otimes 1\right]_{H^{\prime}, H^{\prime}}
$$

(note that $H^{\prime} \subseteq K^{\prime}$ because $K \subseteq H$ ), which are mapped under the isomorphim (4.2) to

$$
r_{H^{\prime}}^{K^{\prime}}, \quad t_{H^{\prime}}^{K^{\prime}}, \quad \lambda_{H^{\prime}, \psi_{H^{\prime}}^{-1}(x)} \quad \text { and } \quad c_{\psi_{H}(\sigma), H^{\prime}}
$$


The isomorphism $\iota: \rho \longrightarrow \rho$ induces a functor

$$
\mathcal{U}: \rho-\bmod \longrightarrow \rho-\bmod , \quad M \longmapsto M^{\prime},
$$

where $M^{\prime}$ is the the module $M$ with $\rho$-action defined by $r \cdot m=\iota(r) m$, for all $m \in M$ and $r \in \rho$. Using the equivalence between $\rho$-mod and $R$-mod, $\mathcal{U}$ can also be written as a functor $\mathcal{U}: R$-mod $\longrightarrow R$-mod, $M \longmapsto M^{\prime}$, where $M^{\prime}$ is the Mackey functor defined by $M^{\prime}(G / H)=M\left(G / H^{\prime}\right)$ and $M^{\prime}(\alpha)=M(\iota(\alpha))$ for all morphisms $\alpha$ in $\mathcal{C}_{R}$.

Proposition 4.15. The functor

$$
\mathcal{U}: \rho-\bmod \longrightarrow \rho-\bmod
$$

$\left(\right.$ or $\mathcal{U}: R$-mod $\longrightarrow R$-mod) is exact and $\mathcal{U}^{2}=i d$.

Proposition 4.16. $\mathcal{U}\left(\rho 1_{H}\right) \simeq \rho 1_{H^{\prime}}$ and $\mathcal{U}\left(R_{G / H}\right) \simeq R_{G / H^{\prime}}$.

Proof. The map $\left.\iota\right|_{\rho 1_{H}}: \rho 1_{H} \longrightarrow \rho 1_{H^{\prime}}$ is an isomorphism from $\left(\rho 1_{H}\right)^{\prime}$ to $\rho 1_{H^{\prime}}$ because $\iota: \rho \longrightarrow \rho$ is an isomorphism of algebras. The second part is equivalent to the first using the equivalence between $R$-modules and $\rho$-modules.

\section{Part II. The proofs}

\section{Preliminaries}

Fix a finite group $G$ and a commutative ring $\mathcal{Z}$ with unit. Let $R$ be the representation functor for $G$ with coefficients in $\mathcal{Z}$ and let $F=F P_{\mathcal{Z}}$ be the fixed point functor defined in Example 1.6. Proposition 2.17 gives the image of the functor $\mathcal{H}_{R}(\cdot, F)$ on the projective $R$-modules $R_{X}$. In this section, we will see what the image is on maps between projective modules and, in particular, what $\mathcal{H}_{R}(\cdot, F)(G / G)$ is. Needless to say, this is what we need to know to compute the derived functor $\operatorname{Ext}_{R}(\cdot, F)$ of $\mathcal{H}_{R}(\cdot, F)$.

As we saw before, the representation functor $R$ is a commutative Green functor and $F$ is a module over $R$. The action of the $\operatorname{ring} R(G / H)=R[H]$ on the $R(G / H)$ module $F(G / H)=\mathcal{Z}$ is given by $\sigma \cdot z=\operatorname{dim}(\sigma) z$ for all representations $\sigma \in R[H]$ and $z \in \mathcal{Z}$. The functor $F$ is also a Green functor since $F(G / H)=\mathcal{Z}$ is a ring for all $H \subseteq G$ and $F(f)=1$ is a ring map for all $G$-maps $f: G / H \longrightarrow G / K$. The maps $F(\widehat{f})$ also satisfy the transfer conditions in Definition 2.4.

Let $\epsilon: R \longrightarrow F$ be the map defined by the usual augmentation map $R[H] \longrightarrow \mathcal{Z}$, $\sigma \longmapsto \operatorname{dim} \sigma$ (tensored with $\mathcal{Z}$ ) at each $G / H$. This is a map of $R$-modules and a map of Green functors. As a map of Green functors, $\epsilon$ induces a map of algebras $\epsilon: \rho \longrightarrow \rho(F)$, where $\rho$ and $\rho(F)$ are the algebras associated to the Green functors $R$ and $F$, respectively. As a map of $R$-modules, since $\epsilon$ is surjective, it gives $F \simeq R / I$, where $I=\operatorname{ker} \epsilon$ is the augmentation ideal. Kernels of maps of Mackey functors are defined levelwise, in this case $I(G / H)=I[H]$ is the usual augmentation ideal of the ring $R[H]$. Still as a map of $R$-modules, $\epsilon$ induces surjective maps $\epsilon_{X}: R_{X} \longrightarrow F_{X}$ for all $G$-sets $X$, and $\operatorname{ker}\left(\epsilon_{X}\right)=(\operatorname{ker} \epsilon)_{X}=I_{X}$.

We will say that a map of $R$-modules $f: R_{G / H} \longrightarrow R_{G / K}$ is determined by $v \in R_{G / K}(G / H)=1_{H} \rho 1_{K}$, if $f$ corresponds to the map of $\rho$-modules $\rho 1_{H} \longrightarrow \rho 1_{K}$ given by multiplication on the right by $v$.

Proposition 5.1. Let

$$
f: R_{G / H} \longrightarrow R_{G / K}
$$


be the map determined by $v \in R_{G / K}(G / H)=1_{H} \rho 1_{K}$. Then the map $\mathcal{H}_{R}(f, R)$ : $R_{G / K} \longrightarrow R_{G / H}$ is determined by $\widehat{v} \in R_{G / H}(G / K)=1_{K} \rho 1_{H}$.

Proof. This proposition is really about the isomorphism

$$
\mathcal{H}_{R}\left(R_{X}, R\right) \stackrel{\varphi}{\longrightarrow} R_{X}
$$

in Proposition 2.17 when $M=R$. At the $G$-set $Y$, by definition of $\mathcal{H}_{R}$

$$
\mathcal{H}_{R}\left(R_{X}, R\right)(Y)=\operatorname{Hom}_{R-\bmod }\left(R_{X}, R_{Y}\right)=\operatorname{Hom}_{\mathcal{C}_{R}}(X, Y)
$$

by (2.3)

$$
R_{X}(Y)=\operatorname{Hom}_{R \text {-mod }}\left(R_{Y}, R_{X}\right)=\operatorname{Hom}_{\mathcal{C}_{R}}(Y, X) .
$$

So $\varphi$ is the opposite map $\mathcal{C}_{R} \stackrel{\simeq}{\longrightarrow} \mathcal{C}_{R}^{o p}$ that sends a morphism $g$ to its transfer $\widehat{g}$.

The proof now should be easy. Let $f^{*}=\mathcal{H}_{R}(f, R)$. At the $G$-set $G / K$, the map $f^{*}(G / K)$ sends the identity map $i d \in \operatorname{Hom}_{R \text {-mod }}\left(R_{G / K}, R_{G / K}\right)$ to $f \in$ $\operatorname{Hom}_{R \text {-mod }}\left(R_{G / H}, R_{G / K}\right)$. By Proposition $2.17 f^{*}: R_{G / K} \longrightarrow R_{G / K}$ is determined by $f^{*}(G / K)(i d)$, which is $f$ identified with $\widehat{v}$ as an element in $R_{G / H}(G / K)=$ $1_{K} \rho 1_{H}$.

Proposition 5.2. Let $f$ be the map on Proposition 5.1, Then $\mathcal{H}_{R}(f, F): F_{G / K} \longrightarrow$ $F_{G / H}$ is the map determined by $\epsilon(\widehat{v})$.

Proof. We already know that $\mathcal{H}_{R}(f, R)$ is $\times \widehat{v}$. Let $f^{*}=\mathcal{H}_{R}(f, F)$. The diagram

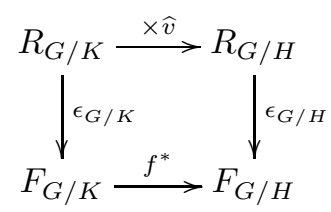

is commutative. It can also be written as
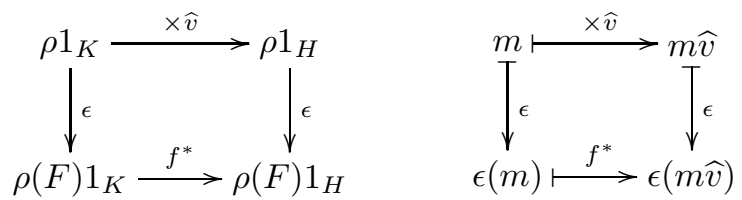

(The bottom row is actually a map of $\rho(F)$-modules but is also a map of $\rho$-modules via the augmentation map $\epsilon: \rho \longrightarrow \rho(F)$.) The vertical arrows are surjective and $\epsilon(m \widehat{v})=\epsilon(m) \epsilon(\widehat{v})$ so $f^{*}$ is $\times \epsilon(\widehat{v})$.

There is an abuse of notation in the statement of Proposition 5.2. The element $\epsilon(\widehat{v}) \in \rho(F)$ determines a map of $F$-modules from $F_{G / K}$ to $F_{G / H}$ which corresponds to the map of $\rho(F)$-modules $\rho(F) 1_{K} \stackrel{x \epsilon(\widehat{v})}{\longrightarrow} \rho(F) 1_{K}$. But since $F$ is an $R$-module, the map $F_{G / K} \stackrel{\times \epsilon(\widehat{v})}{\longrightarrow} F_{G / H}$ is also a map of $R$-modules. We will write $\times \epsilon(\widehat{v})$, or even just $\times \widehat{v}$, to denote the map of $R$-modules too, as we did in Proposition 5.2 .

Let $X$ be a transitive $G$-set. The functor $F_{X}$ is representable as an $F$-module. As an $R$-module, $F_{X}$ is a quotient of a representable $R$-module, $F_{X} \simeq R_{X} / I_{X}$. In terms of modules over the algebra $\rho$, the corresponding module $\widetilde{F}_{X}$ is a quotient of the submodule of $\widetilde{R}_{X}$ of $\rho$. The units $1_{R}$ and $1_{F}$ of the Green functors $R$ and 
$F$ induce maps of algebras $\mu \stackrel{1_{R}}{\longrightarrow} \rho$ and $\mu \stackrel{1_{F}}{\longrightarrow} \rho(F)$. It is easy to check that $1_{F}$ is surjective and it factors throuth $\rho$, i.e. the following diagram commutes:

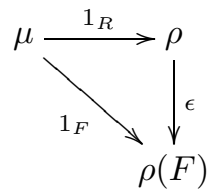

Since $1_{F}$ is surjective, the elements $\lambda_{H, a}$ in $\rho(F)$ can be written in terms of $r_{K}^{H}, t_{K}^{H}$ and $c_{x, H}$ which are in $\mu$ and also in $\rho$. By abuse of notation, we will consider $\widetilde{F}_{X}$ as a submodule of $\rho$ since we do not need any of the extra generators $\lambda_{H, a} \in \rho(F)$, and drop any references to the algebra $\rho(F)$, unless it is needed to clarify some argument.

Let $\widetilde{F}$ be the $\rho$-module corresponding to $F$. Then $\widetilde{F}=\bigoplus_{H \subseteq G} \mathcal{Z}$ with $\rho$-action defined by: $r_{K}^{H}$ sends the $H$-th to the $K$-th component, $t_{K}^{H}$ is multiplication by $[H: K]$ from the $K$-th to the $H$-th component, $c_{x, H}$ sends the $H$-th component to the ${ }^{x} H$-th component, and $\lambda_{H, \sigma}$ is multiplication by $\operatorname{dim}(\sigma)$ in the $H$-th component. So $\widetilde{F}$ is isomorphic the submodule generated by the elements $r_{H}^{G}$ with $H \subseteq G$. We will denote this module by $\mathcal{Z}\left[r_{H}^{G}: H \subseteq G\right]$.

Proposition 5.3. (i) $\operatorname{Hom}_{\rho}\left(\rho 1_{H}, \widetilde{F}\right)=\mathcal{Z}$ is generated as a $\mathcal{Z}$-module by $1_{H} \longmapsto$ $r_{H}^{G}$,

(ii) $\operatorname{Hom}_{\rho}\left(r_{K}^{H}, \widetilde{F}\right)=1 \quad \forall K \subseteq H \subseteq G$,

(iii) $\operatorname{Hom}_{\rho}\left(t_{K}^{H}, \widetilde{F}\right)=[H: K] \quad \forall K \subseteq H \subseteq G$,

(iv) $\operatorname{Hom}_{\rho}\left(c_{x, H}, \widetilde{F}\right)=1 \quad \forall x \in G, H \subseteq G$,

(v) $\operatorname{Hom}_{\rho}\left(\lambda_{H, \sigma}, \widetilde{F}\right)=\operatorname{dim} \sigma \quad \forall H \subseteq G, \sigma \in R[H]$,

where $r_{K}^{H}: \rho 1_{K} \longrightarrow \rho 1_{H}, t_{K}^{H}: \rho 1_{H} \longrightarrow \rho 1_{K}, c_{x, H}: \rho 1_{H^{x}} \longrightarrow \rho 1_{H}$ and $\lambda_{H, \sigma}:$ $\rho 1_{H} \longrightarrow \rho 1_{H}$ denote multiplication on the right by $r_{K}^{H}, t_{K}^{H}, c_{x, H}$ and $\lambda_{H, \sigma}$, respectively.

Proof. A morphism of $\rho$-modules $\rho 1_{H} \longrightarrow \widetilde{F}$ is determined by the image of $1_{H}$. Since $\widetilde{F}=\mathcal{Z}\left[r_{H}^{G}: H \subseteq G\right]$, the $\mathcal{Z}$-module $\operatorname{Hom}_{\rho}\left(\rho 1_{H}, \widetilde{F}\right)$ is generated by the maps $\varphi_{K}: 1_{H} \longmapsto r_{K}^{G}$. But

$$
\varphi_{K}\left(1_{H}\right)=\varphi_{K}\left(1_{H}^{2}\right)=1_{H} \varphi_{K}\left(1_{H}\right)=1_{H} r_{K}^{G}=\left\{\begin{array}{cc}
0 & \text { if } K \neq H, \\
r_{H}^{G} & \text { if } K=H
\end{array}\right.
$$

so $\varphi_{H}$ generates $\operatorname{Hom}_{\rho}\left(\rho 1_{H}, \widetilde{F}\right)$.

Parts (ii), (iii), (iv) and (v) follow from part (i) and the remark before the lemma.

Proposition 5.4. If $K \subseteq H$ and $K \neq H$, then $t_{K}^{H} r_{K}^{H}$ acts as multiplication by $[H: K]$ on the $\rho$-module $\widetilde{F}_{G / L}$ for all $L \subseteq G$.

Proof. To make this proof precise, we need to go back to the algebra $\rho(F)$. By (2.1g) $t_{K}^{H} r_{K}^{H}=\lambda_{H, t_{K}^{H}(1)}$ in $\rho(F)$, where $t_{K}^{H}(1)$ is notation for $F\left(\widehat{\pi}_{K}^{H}\right)(1)$ and $\pi_{K}^{H}$ : $G / K \longrightarrow G / H$ is the quotient map. Since $F\left(\widehat{\pi}_{K}^{H}\right)(1)=[H: K]$, the result follows from (2.1C) and from the fact that the action of $\rho$ on $\widetilde{F}_{G / L}$ is induced by the action of $\rho(F)$ on $\rho(F) 1_{L}$. 
Let $\widetilde{\epsilon}: \rho 1_{H} \longrightarrow \widetilde{F}$ be the map of $\rho$-modules corresponding to $\epsilon: R \longrightarrow F$. It is defined by $1_{G} \longmapsto r_{G}^{G}=1_{G}$. Recall that $I=\operatorname{ker} \epsilon$ is the augmentation ideal. So $\widetilde{I}$ is the kernel of $\widetilde{\epsilon}$.

Lemma 5.5. Suppose $G$ is abelian and is generated by $\left\{g_{1}, \ldots, g_{n}\right\}$. Then

$$
\widetilde{I}=\rho\left\{\lambda_{G, \sigma_{i}}-1_{G} \mid i=1, \ldots, n\right\},
$$

where $\sigma_{i}=\varphi\left(g_{i}\right)$ for some fixed isomorphism $\varphi: \mathcal{Z}[G] \longrightarrow R[G]$.

Proof. Since the map $r_{H}^{G}: R[G] \longrightarrow R[H]$ is surjective because $G$ is abelian, then $I[H]=r_{H}^{G}(I[G])$, and this implies that $\widetilde{I}$ is generated by $1_{G} \widetilde{I}=I[G]$. The ideal $I[G]$ is generated by $\{\sigma-1 \mid \sigma$ irreducible character $\}$. Since $R[G]$ is isomorphic to the group ring $\mathcal{Z}[G]$, the characters $\sigma_{i}$ generate $R[G]$ and $\sigma_{i}-1$ generate $I[G]$ as an $R[G]$-module.

Corollary 5.6. Let $\partial_{0}:\left(\rho 1_{G}\right)^{n} \longrightarrow \rho 1_{G}$ be the map

$$
\partial_{0}=\left[\begin{array}{lll}
\lambda_{G, \sigma_{1}}-1_{G} & \ldots & \lambda_{G, \sigma_{n}}-1_{G}
\end{array}\right],
$$

where $\sigma_{i}$ was defined in Lemma 5.5 Then

$$
0 \longleftarrow \widetilde{F} \stackrel{\epsilon}{\longleftarrow} \rho 1_{G} \stackrel{\partial_{0}}{\longleftarrow}\left(\rho 1_{G}\right)^{n}
$$

is exact.

Another way of stating this corollary is

$$
\widetilde{F}=\rho 1_{G} / \rho\left\{\lambda_{G, \sigma_{i}}-1_{G} \mid i=1, \ldots, n\right\} .
$$

In general

Corollary 5.7. If $G$ is abelian, then

$$
\widetilde{F}_{G / H}=\rho 1_{H} / \rho\left\{\lambda_{H, \sigma}-1_{H} \mid \sigma \in R[H] \text { irreducible }\right\}
$$

for all $H \subseteq G$.

Proof. Since $\epsilon: R \longrightarrow F$ is surjective, $\epsilon_{G / H}: R_{G / H} \longrightarrow F_{G / H}$ is also. As in Lemma 5.5, ker $\epsilon_{G / H}$ is generated by $\operatorname{ker} \epsilon_{G / H}(G / H)$ which is generated by $\lambda_{H, \sigma}-1_{H}$.

Let $G=(\mathbb{Z} / 2)^{n}$ and $\mu$ be the Mackey algebra for $G$.

Proposition 5.8. If $G=(\mathbb{Z} / 2)^{n}$, the algebra $\rho$ is generated by

$$
\left\{t_{K}^{H}, r_{K}^{H} \mid K \subseteq H \subseteq G\right\} .
$$

Proof. If $\sigma_{K}$ is an irreducible representation (or character) of $H \subseteq G$ with ker $=K$, then $[H: K]=2$ and $t_{K}^{H}(1)=1+\sigma_{K}$, where 1 is the trivial representation. Equation (2.1g) with $a=1$ gives

$$
\lambda_{H, \sigma_{K}}=t_{K}^{H} r_{K}^{H}-1_{H} .
$$

Given any representation $\sigma$ of the subgroup $H$, it can be written as a sum of irreducible representations and so, applying (2.1a) and (5.1), we can write $\lambda_{H, \sigma}$ as a linear combination of products of inductions and restrictions.

The relation (1.2g) with $L=K$ and $H=\langle K, x\rangle$ gives

$$
c_{x, K}=r_{K}^{\langle K, x\rangle} t_{K}^{\langle K, x\rangle}-1_{K}
$$

for all $x \in G / H, x \notin H$. 
Corollary 5.9. Let $\pi: \mu \longrightarrow \rho$ be the map that sends any element of $\mu$ to the element in $\rho$ with the same name. Then $\pi$ is surjective and $\rho \simeq \mu / \operatorname{ker} \pi$.

Corollary 5.10. Let $G=(\mathbb{Z} / 2)^{n}=\left\langle T_{1}, \ldots, T_{n}\right\rangle$ and $\mathcal{Z}=\mathbb{F}_{2}$. Let $\partial_{0}:\left(\rho 1_{G}\right)^{n} \longrightarrow$ $\rho 1_{G}$ be

Then

$$
\partial_{0}=\left[\begin{array}{lll}
t_{\left\langle T_{1}\right\rangle^{\prime}}^{G} r_{\left\langle T_{1}\right\rangle^{\prime}}^{G} & \cdots & t_{\left\langle T_{n}\right\rangle^{\prime}}^{G}, r_{\left\langle T_{n}\right\rangle^{\prime}}^{G}
\end{array}\right]
$$

$$
0 \longleftarrow \widetilde{F} \stackrel{\epsilon}{\longleftarrow} \rho 1_{G} \stackrel{\partial_{0}}{\longleftarrow}\left(\rho 1_{G}\right)^{n}
$$

is exact.

Proof. Trivial from Lemma 5.5 and (5.1).

\section{The CYClic CASE}

In this section $G=\mathbb{Z} / 2^{n}=\langle T\rangle$ and the coefficient ring is $\mathbb{F}_{2}$. Let $R$ be the representation functor and $F$ be the fixed point functor as in Section 5 , We prove the following theorem.

Theorem 6.1. The Green functor $\operatorname{Ext}_{R}(F, F)$ is given by

$$
\operatorname{Ext}_{R}(F, F)(G / H)=\mathbb{F}_{2}\left[y_{3}, x_{4}\right] /\left(y_{3}^{2}=0\right)
$$

for $H \subseteq G, H \neq e$, where $x_{4}$ has degree 4 and $y_{3}$ has degree 3 , and

$$
\operatorname{Ext}_{R}(F, F)(G / e)=\mathbb{F}_{2} .
$$

If $K \neq e$, the restriction map $r_{K}^{H}$ is the ring map that sends $y_{3}$ to zero and $x_{4}$ to $x_{4}$, the induction map $t_{K}^{H}$ sends $y_{3} x_{4}^{i}$ to $y_{3} x_{4}^{i}$ and $x_{4}^{i}$ to zero, for all $i \geq 0$.

Let $\rho$ be the algebra associated to $R$. Fix an isomorphism $\varphi: G^{*} \longrightarrow G$ and let $\varphi(\sigma)=T$. The upside down functor $\mathcal{U}$ defined in Section 4.2 switches the maps $\times c_{T, e}$ and $\times \lambda_{G, \sigma}$. We also denote by $T$ the generators of the subgroups $\mathbb{Z} / 2^{k} \subseteq \mathbb{Z} / 2^{n}$ and by $\sigma$ the restriction of $\sigma$ to $\mathbb{Z} / 2^{k}$. So $\mathcal{U}$ switches $c_{T, H}$ with $\lambda_{H^{\prime}, \sigma}$ for all $H \subseteq G$. Also, we will drop the $\times$ on the maps $\times v$. As a map, an element $v$ of $\rho$ always denotes multiplication on the right.

Proposition 6.2. The long exact sequence

$$
R \stackrel{1_{G}+\lambda_{G, \sigma}}{\longleftarrow} R \stackrel{r_{e}^{G}}{\longleftarrow} R_{G / e} \stackrel{1_{e}+c_{T, e}}{\longleftarrow} R_{G / e} \stackrel{t_{e}^{G}}{\longleftarrow} R \stackrel{1_{G}+\lambda_{G, \sigma}}{\longleftarrow} \cdots
$$

is a projective $R$-resolution for $F$.

Proof. The first differential $\partial_{0}=1_{G}+\lambda_{G, \sigma}$ comes from Corollary 5.6. Levelwise, i.e. at the $G$-set $G / H$ with $H=\mathbb{Z} / 2^{k}, \partial_{0}(G / H)$ is $R[H] \stackrel{1+\sigma}{\longrightarrow} R[H]$ and its kernel is generated by the regular representation $1+\sigma+\cdots+\sigma^{2^{k}-1}=t_{e}^{H}(1)$. This implies that ker $\partial_{0}$ is generated as an $R$-module by the trivial representation of $R(G / e)=R[e]$ and so the next term of the resolution is $R_{G / e}$ and the differential $\partial_{1}$ is $r_{e}^{G}$.

It looks like $\partial_{1}$ should be $t_{e}^{G}$ and not $r_{e}^{G}$, but recall that the elements of $\rho$ act on the left as the morphisms in an $R$-module and act on the right to define maps between representable $R$-modules (or $\rho$-submodules). Also, the trivial representation of the trivial subgroup lies in $R(G / e)=1_{e} \rho 1_{G}$ and corresponds to the element $r_{e}^{G}$, and so $\operatorname{ker} \partial_{0}=\rho r_{e}^{G}$.

At $G / H$ with $H=\mathbb{Z} / 2^{k}, \partial_{1}(G / H)$ is the map $R[e]^{2^{n-k}} \longrightarrow R[H]$ that sends 1 of each component to $1+\sigma+\cdots+\sigma^{2^{k}-1}$. The kernel is generated by the elements 
$(1,1,0, \ldots, 0),(0,1,1,0, \ldots, 0), \ldots,(0, \ldots, 0,1,1)$. Again all of them are obtained from the kernel of $\partial_{1}(G / e)$, and all of the elements of ker $\partial_{1}(G / e)$ are obtained from $(1,1,0, \ldots, 0)$ by conjugation. Since $(1,1,0, \ldots, 0)$ in $R_{G / e}(G / e)=1_{e} \rho 1_{e}$ corresponds to $1_{e}+c_{T, e}$, ker $\partial_{1}=\rho\left(1_{e}+c_{T, e}\right)$, and so the next term of the resolution is $R_{G / e}$ with differential $\partial_{2}=1_{e}+c_{T, e}$.

Now notice that $\mathcal{U}\left(\operatorname{ker} \partial_{1}\right)=\mathcal{U}\left(\rho\left(1_{e}+c_{T, e}\right)\right)=\rho\left(1_{G}+\lambda_{G, \sigma}\right)$ and, by Lemma 5.5, this is the augmentation ideal. To get the rest of the resolution, including $\partial_{2}$, just apply the upside down functor $\mathcal{U}$ to the bit of the resolution we already have: $\partial_{2}=\mathcal{U}\left(\partial_{0}\right), \partial_{3}=\mathcal{U}\left(\partial_{1}\right), \partial_{4}=\mathcal{U}\left(\partial_{2}\right)=\mathcal{U}^{2}\left(\partial_{0}\right)=\partial_{0}$ and we are back to the beginning.

We constructed the resolution with both $R$-modules and $\rho$-modules. These two categories are equivalent and sometimes one of them is better than the other. The functors have the advantage of breaking the computation of kernels to each level, and the algebra $\rho$ provides a good way to put everything together and identify the maps more easily, since we are dealing with submodules of $\rho$. We can also write the resolution (6.3) as

$$
\rho 1_{G} \stackrel{1_{G}+\lambda_{G, \sigma}}{\longleftarrow} \rho 1_{G} \stackrel{r_{e}^{G}}{\longleftarrow} \rho 1_{e} \stackrel{1_{e}+c_{T, e}}{\longleftarrow} \rho 1_{e} \stackrel{t_{e}^{G}}{\longleftarrow} \rho 1_{G} \stackrel{1_{G}+\lambda_{G, \sigma}}{\longleftarrow} \cdots .
$$

Let $M$ be the Mackey functor defined by $M(G / H)=\mathcal{Z}$ if $H \neq e$ and $M(G / e)=$ $0, M(f)=1$ for all $G$-maps $f: G / K \longrightarrow G / H$ if $K \neq e$ and all transfers $M(\widehat{f})=0$. Let $N$ be the $R$-module that has the same value on objects as $M$ with morphisms $N\left(\pi_{K}^{H}\right)=0, N\left(\gamma_{x, H}\right)=1$ and $N\left(\widehat{\pi}_{K}^{H}\right)=1$, where $\pi_{K}^{H}: G / K \longrightarrow G / H$ is the quotient map and $\gamma_{x, H}: G / H \longrightarrow G / H$ is a conjugation map.

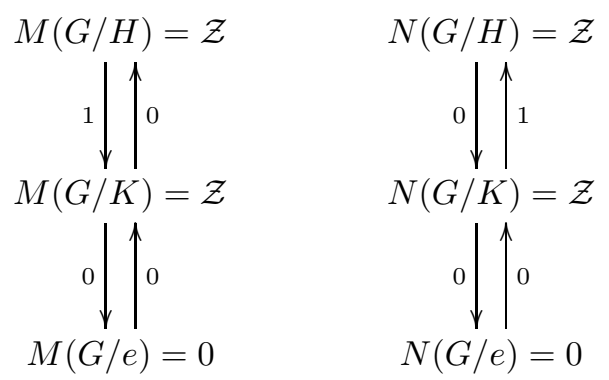

\section{Proposition 6.3.}

$$
\operatorname{Ext}_{R}^{n}(F, F)= \begin{cases}F & \text { if } n=0 \\ 0 & \text { if } n=1+4 k \text { or } n=2+4 k, k \geq 0 \\ N & \text { if } n=3+4 k, k \geq 0 \\ M & \text { if } n=4 k, k>0 .\end{cases}
$$

Proof. Since the resolution (6.3) is 4-periodic, $\operatorname{Ext}_{R}^{i+4}(F, F)=\operatorname{Ext}_{R}^{i}(F, F)$ for all $i \geq 1$.

Apply the functor $\mathcal{H}_{R}(\cdot, F)$ to the resolution (6.3). By Proposition 2.17 we get the complex

$$
F \stackrel{\delta_{0}}{\longrightarrow} F \stackrel{\delta_{1}}{\longrightarrow} F_{G / e} \stackrel{\delta_{2}}{\longrightarrow} F_{G / e} \stackrel{\delta_{3}}{\longrightarrow} F \stackrel{\delta_{0}}{\longrightarrow} F \stackrel{\delta_{1}}{\longrightarrow} \cdots,
$$

where $\delta_{i}=\mathcal{H}_{R}\left(\partial_{i}, F\right)$. From Proposition 5.2 we have $\delta_{0}=1_{G}+\lambda_{G, \sigma}, \delta_{1}=t_{e}^{G}, \delta_{2}=$ $1_{e}+c_{T, e}$ and $\delta_{3}=r_{e}^{G}$. Since $\operatorname{dim}(\sigma)=1, \delta_{0}=0$, and so $\operatorname{Ext}_{R}^{0}(F, F)=\operatorname{ker} \delta_{0}=F$. 
A map of $R$-modules from a functor $F_{G / K}$ to any module is determined at the $G$-set $G / K$. So $\delta_{1}, \delta_{2}$ and $\delta_{3}$ are determined by $\delta_{1}(G / G), \delta_{2}(G / e)$ and $\delta_{3}(G / e)$. Writing the differentials in matrix form, instead of using an element of $\rho$, the complex becomes

$$
\begin{aligned}
& \text { at } G / G: \mathbb{F}_{2} \stackrel{0}{\longrightarrow} \mathbb{F}_{2} \stackrel{1}{\longrightarrow} \mathbb{F}_{2} \stackrel{0}{\longrightarrow} \mathbb{F}_{2} \stackrel{0}{\longrightarrow} \mathbb{F}_{2} \stackrel{0}{\longrightarrow} \cdots, \\
& \text { at } G / H: \mathbb{F}_{2} \stackrel{0}{\longrightarrow} \mathbb{F}_{2} \stackrel{\Delta}{\longrightarrow} \mathbb{F}_{2}^{2^{n-k}} \stackrel{A}{\longrightarrow} \mathbb{F}_{2}^{2^{n-k}} \stackrel{0}{\longrightarrow} \mathbb{F}_{2} \stackrel{0}{\longrightarrow} \cdots, \\
& \text { at } G / e: \mathbb{F}_{2} \stackrel{0}{\longrightarrow} \mathbb{F}_{2} \stackrel{\Delta}{\longrightarrow} \mathbb{F}_{2}^{2^{n}} \stackrel{A}{\longrightarrow} \mathbb{F}_{2}^{2^{n}} \stackrel{\epsilon}{\longrightarrow} \mathbb{F}_{2} \stackrel{0}{\longrightarrow} \cdots,
\end{aligned}
$$

where $H=\mathbb{Z} / 2^{k}$ for $1<k<n, \Delta$ is the diagonal map and

$$
A=\left[\begin{array}{ccccc}
1 & 0 & \cdots & 0 & 1 \\
1 & 1 & \ddots & \vdots & 0 \\
0 & 1 & \ddots & 0 & \vdots \\
\vdots & \ddots & \ddots & 1 & 0 \\
0 & \cdots & 0 & 1 & 1
\end{array}\right]
$$

Now compute the cohomology of each row to get $\operatorname{Ext}_{R}^{n}(F, F)$ at each transitive $G$-set. The morphisms are induced from $F$ and $F_{G / e}$ since each $\operatorname{Ext}_{R}^{n}(F, F)$ is a subfunctor of one of these.

Proposition 6.4. As an algebra, $\operatorname{Ext}_{\rho}(\widetilde{F}, \widetilde{F})=\mathbb{F}_{2}\left[y_{3}, x_{4}\right] /\left(y_{3}^{2}=0\right)$, where $x_{4}$ has degree 4 and $y_{3}$ has degree 3.

Proof. As a graded vector space, $\operatorname{Ext}_{\rho}(\widetilde{F}, \widetilde{F})=\operatorname{Ext}_{R}(F, F)(G / G)$. The algebra structure is given by composition of chain maps since $\operatorname{Ext}_{\rho}^{i}(\widetilde{F}, \widetilde{F})=\left[P_{*}, P[-i]_{*}\right]$, where $P_{*}$ is the resolution (6.4).

Let $x_{4}$ and $y_{3}$ be the nonzero classes in $\operatorname{Ext}_{\rho}(\widetilde{F}, \widetilde{F})$ of degree 3 and 4 , respectively. Let $\alpha_{*}: P_{*} \longrightarrow P[-4]_{*}$ be

$$
\alpha_{i}= \begin{cases}0, & 0 \leq i \leq 3 \\ 1, & i \geq 4\end{cases}
$$

and $\beta_{*}: P_{*} \longrightarrow P[-3]_{*}$ be

$$
\beta_{i}=\left\{\begin{array}{cl}
0 & \text { if } 0 \leq i \leq 2, \\
r_{e}^{G} & \text { if } i=3+4 k, k \geq 0, \\
\sum_{j \text { even }} \lambda_{G, \sigma^{j}} & \text { if } i=4+4 k, k \geq 0, \\
t_{e}^{G} & \text { if } i=5+4 k, k \geq 0, \\
\sum_{j \text { even }} c_{T^{j}, e} & \text { if } i=6+4 k, k \geq 0 .
\end{array}\right.
$$

It is easy to see that both $\alpha_{*}$ and $\beta_{*}$ are chain maps. To see that they are not chain homotopic to zero, we are going to apply Lemma 3.2.

The chain map $\alpha_{*}$ is chain homotopic to zero if and only if there is a map $\phi: \rho 1_{e} \longrightarrow \widetilde{F}$ such that $\phi \partial_{3}=\epsilon \alpha_{4}$, where $\epsilon: \rho 1_{G} \longrightarrow \widetilde{F}$ is the augmentation map. 
A map $\phi: \rho 1_{e} \longrightarrow \widetilde{F}$ is either $r_{e}^{G}$ or zero. So $\phi \partial_{3}$ is $1_{G}+\lambda_{G, \sigma}$ or zero, which is always different from $\epsilon \alpha_{4}=1_{G}$.

Again by Lemma 3.2, the chain map $\beta_{*}$ is chain homotopic to zero if and only if there is a map $\phi: \rho 1_{e} \longrightarrow \widetilde{F}$ such that $\phi \partial_{2}=\epsilon \beta_{3}=r_{e}^{G}$. As above, a nonzero map $\varphi$ must be $r_{e}^{G}$, so $\phi \partial_{2}=\left(1_{e}+c_{T, e}\right) r_{e}^{G}=0 \neq \epsilon \beta_{3}$.

Since $\alpha_{*}$ and $\beta_{*}$ are not chain homotopic to zero, they represent the classes $x_{4}$ and $y_{3}$.

The class $y_{3}^{2}$ is zero because $\operatorname{Ext}_{\rho}^{6}(\widetilde{F}, \widetilde{F})=0$.

Composing any chain map $\gamma: P_{*} \longrightarrow P[-n]_{*}$ with $\alpha_{*}$, on the left or on the right, gives

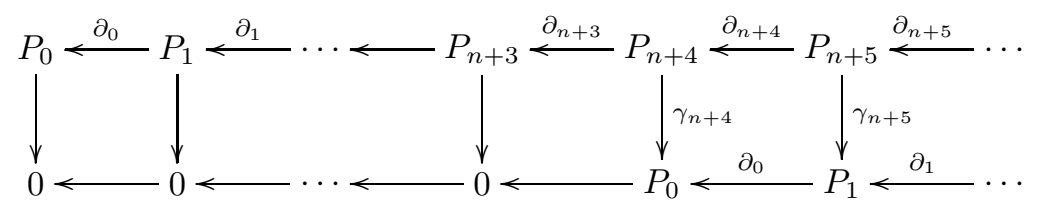

Since the resolution $P_{*}$ is 4 -periodic, i.e. $\partial_{i+4}=\partial_{i}$ for all $i \geq 0$, we can assume that $\gamma_{*}$ satisfies $\gamma_{i+4}=\gamma_{i}$ for $i \geq n$ (if not, we can replace $\gamma_{*}$ by a chain homotopic map satisfying this condition). So, by Lemma [3.2, if $\gamma_{*}$ is not chain homotopic to zero, $\alpha_{*} \gamma_{*}=\gamma_{*} \alpha_{*}$ is not either. This argument implies that $\alpha_{*}^{j}$ and $\beta_{*} \alpha_{*}^{j}$ are not chain homotopic to zero for all $j \geq 0$, and since all these classes have different degrees, they are linearly independent.

Propositions 6.3 and 6.4 together imply Theorem 6.1

\section{THE $\mathbb{Z} / 2 \times \mathbb{Z} / 2$ CASE}

Let $G=\mathbb{Z} / 2 \times \mathbb{Z} / 2$ and let the coefficient ring be $\mathbb{F}_{2}$. The goal of this section is to compute the graded Green functor $\operatorname{Ext}_{R}(F, F)$, where $R$ is the representation Green functor and $F$ is the fixed point functor, a module over $R$. First we build a projective resolution. The tools used are the short exact sequence trick (Proposition 3.1), the upside down functor $\mathcal{U}$ defined in Section 4.2 and the induction functor $\operatorname{Ind}_{H}^{G}$ of Section 4.1. Then we compute the algebra $\operatorname{Ext}_{R}(F, F)(G / G)$. The value of $\operatorname{Ext}_{R}(F, F)$ at the other orbits and morphisms are obtained by applying the restriction functor $\operatorname{Res}_{H}^{G}$.

7.1. A projective resolution. Let $\mu$ be the Mackey algebra over $\mathbb{F}_{2}$ for the group $\mathbb{Z} / 2 \times \mathbb{Z} / 2$. Let $\rho$ be the algebra associated to $R$. Let $S$ and $T$ be two generators of the group $G$. We will also denote by $S, T$ and $S T$ the subgroups of order two generated by these elements.

Proposition 7.1. $\rho=\mu /\left(t_{e}^{G} r_{e}^{G}+t_{S}^{G} r_{S}^{G}+t_{T}^{G} r_{T}^{G}+t_{S T}^{G} r_{S T}^{G}\right)$.

Proof. By Corollary 5.9 all we need to do is to compute $\operatorname{ker} \pi$.

The rank of $\mu$ is $r k A\left(\Omega^{2}\right)=53$, where $\Omega=\amalg_{H \subseteq G} G / H$. The rank of $\rho$ is $\operatorname{rkR}\left(\Omega^{2}\right)=52$. So ker $\pi$ has rank 1 .

Equation (2.1g) with $H=G, K=e$ and $a=1$ gives

$$
t_{e}^{G} r_{e}^{G}=\lambda_{G, 1+\sigma_{S}+\sigma_{T}+\sigma_{S T}}
$$

where $\sigma_{L}$ denotes the irreducible character with kernel $L$. Now apply (5.1) and (2.1a) to get

$$
t_{e}^{G} r_{e}^{G}=t_{S}^{G} r_{S}^{G}+t_{T}^{G} r_{T}^{G}+t_{S T}^{G} r_{S T}^{G}
$$


Notice that the relation (7.1) does not hold in $\mu$ because the four elements involved are linearly independent in $\mu$ (see [7]), so ker $\pi$ is generated by $t_{e}^{G} r_{e}^{G}+t_{S}^{G} r_{S}^{G}+t_{T}^{G} r_{T}^{G}+$ $t_{S T}^{G} r_{S T}^{G}$.

Lemma 7.2. The sequences

$$
\rho 1_{H} \stackrel{t_{e}^{H} r_{e}^{H}}{\longleftarrow} \rho 1_{H} \stackrel{r_{e}^{H}}{\longleftarrow} \rho 1_{e} \stackrel{r_{e}^{H} t_{e}^{H}}{\longleftarrow} \rho 1_{e} \stackrel{t_{e}^{H}}{\longleftarrow} \rho 1_{H} \stackrel{t_{e}^{H} r_{e}^{H}}{\longleftarrow} \cdots
$$

for $H=S, T, S T$, are exact.

Proof. Apply the functor $\operatorname{Ind}_{H}^{G}$ to the resolution (6.4) with $G$ replaced by $H$. The sequence obtained is exact because $\operatorname{Ind}_{H}^{G}$ is an exact functor.

Lemma 7.3. The sequences

$$
\rho 1_{H} \stackrel{r_{H}^{G} t_{H}^{G}}{\longleftarrow} \rho 1_{H} \stackrel{t_{H}^{G}}{\longleftarrow} \rho 1_{G} \stackrel{t_{H}^{G} r_{H}^{G}}{\longleftarrow} \rho 1_{G} \stackrel{r_{H}^{G}}{\longleftarrow} \rho 1_{H} \stackrel{r_{H}^{G} t_{H}^{G}}{\longleftarrow} \cdots
$$

for $H=S, T, S T$, are exact.

Proof. Apply the upside down functor $\mathcal{U}$ to Lemma 7.2

Let $M=R_{G / S} \oplus R_{G / T} \oplus R_{G / S T}$ and let $\widetilde{M}$ be the $\rho$-module corresponding to $M$.

Lemma 7.4. Let $\partial_{0}: \rho 1_{G}^{2} \longrightarrow \rho 1_{G}, \partial_{1}: \widetilde{M} \longrightarrow \rho 1_{G}^{2}$ and $\partial_{2}: \widetilde{M} \oplus \rho 1_{e} \longrightarrow \widetilde{M}$ be the maps

$$
\partial_{0}=\left[\begin{array}{ll}
t_{S}^{G} r_{S}^{G} & t_{T}^{G} r_{T}^{G}
\end{array}\right], \quad \partial_{1}=\left[\begin{array}{ccc}
r_{S}^{G} & 0 & r_{S T}^{G} \\
0 & r_{T}^{G} & r_{S T}^{G}
\end{array}\right]
$$

and

$$
\partial_{2}=\left[\begin{array}{cccc}
r_{S}^{G} t_{S}^{G} & 0 & 0 & r_{e}^{S} \\
0 & r_{T}^{G} t_{T}^{G} & 0 & r_{e}^{T} \\
0 & 0 & r_{S T}^{G} t_{S T}^{G} & r_{e}^{S T}
\end{array}\right],
$$

where an entry $m \in \rho$ is a map $\rho 1_{K} \stackrel{\times m}{\longleftarrow} \rho 1_{H}$. Then

$$
\rho 1_{G} \stackrel{\partial_{0}}{\longleftarrow} \rho 1_{G}^{2} \stackrel{\partial_{1}}{\longleftarrow} \widetilde{M} \stackrel{\partial_{2}}{\longleftarrow} \widetilde{M} \oplus \rho 1_{e}
$$

is exact.

Proof. $\operatorname{Im} \partial_{i+1} \subseteq$ ker $\partial_{i}, i=0,1:$ Trivial.

$\operatorname{ker} \partial_{0} \subseteq \operatorname{Im} \partial_{1}$ : The elements in ker $\partial_{0}$ are of the form $\left(v_{1}, 0\right)$ with $v_{1} \in \operatorname{ker} t_{S}^{G} r_{S}^{G}$, or $\left(0, v_{2}\right)$ with $v_{2} \in \operatorname{ker} t_{T}^{G} r_{T}^{G}$, or $\left(v_{1}, v_{2}\right)$ with $v_{1} \notin \operatorname{ker} t_{S}^{G} r_{S}^{G}, v_{2} \notin \operatorname{ker} t_{T}^{G} r_{T}^{G}$ and $v_{1} t_{S}^{G} r_{S}^{G}=v_{2} t_{T}^{G} r_{T}^{G}$. The first case gives $\rho\left(r_{S}^{G}, 0\right) \subseteq \operatorname{ker} \partial_{0}$, the second case gives $\rho\left(0, r_{T}^{G}\right) \subseteq \operatorname{ker} \partial_{0}$, and if $\left(v_{1}, v_{2}\right)$ is in the third case, then both $v_{1} t_{T}^{G} r_{T}^{G}$ and $v_{2} t_{S}^{G} r_{S}^{G}$ belong to

$$
\rho t_{S}^{G} r_{S}^{G} \cap \rho t_{T}^{G} r_{T}^{G}=\mathbb{F}_{2}\left\langle t_{e}^{G} r_{e}^{G}, t_{e}^{S T} r_{e}^{G}\right\rangle=\rho t_{e}^{S T} r_{e}^{G}
$$

and so $\left(v_{1}, v_{2}\right) \in \rho\left(r_{S T}^{G}, r_{S T}^{G}\right)$ because $t_{e}^{S T} r_{e}^{G}=r_{S T}^{G}\left(t_{T}^{G} r_{T}^{G}\right)=r_{S T}^{G}\left(t_{S}^{G} r_{S}^{G}\right)$.

ker $\partial_{1} \subseteq \operatorname{Im} \partial_{2}$ : The element $\left(v_{1}, v_{2}, v_{3}\right) \in \widetilde{M}$ is in ker $\partial_{1}$ iff

$$
v_{1} r_{S}^{G}=v_{2} r_{T}^{G}=v_{3} r_{S T}^{G} .
$$

Obviously $\left(v_{1}, 0,0\right),\left(0, v_{2}, 0\right)$ and $\left(0,0, v_{3}\right)$ are in the kernel of $\partial_{1}$ if $v_{1} \in \operatorname{ker} r_{S}^{G}=$ $\rho r_{S}^{G} t_{S}^{G}, v_{2} \in \operatorname{ker} r_{T}^{G}=\rho r_{T}^{G} t_{T}^{G}$ and $v_{3} \in \operatorname{ker} r_{S T}^{G}=\rho r_{S T}^{G} t_{S T}^{G}$. 
Now suppose that $\left(v_{1}, v_{2}, v_{3}\right) \in \operatorname{ker} \partial_{1}$ with $v_{1} \notin \operatorname{ker} r_{S}^{G}$. Then $v_{2} \notin \operatorname{ker} r_{T}^{G}$ and $v_{3} \notin \operatorname{ker} r_{S T}^{G}$. We want to show that

$$
\left(v_{1}, v_{2}, v_{3}\right) \in \rho\left(r_{e}^{S}, r_{e}^{T}, r_{e}^{S T}\right) \bmod \rho\left(r_{S}^{G} t_{S}^{G}, 0,0\right) \oplus \rho\left(0, r_{T}^{G} t_{T}^{G}, 0\right) \oplus \rho\left(0,0, r_{S T}^{G} t_{S T}^{G}\right) .
$$

Since

$$
v_{1} r_{S}^{G} \in \rho r_{T}^{G} \cap \rho r_{S T}^{G}=\mathbb{F}_{2}\left\langle r_{e}^{G}, t_{e}^{G} r_{e}^{G}, t_{e}^{S} r_{e}^{G}, t_{e}^{T} r_{e}^{G}, t_{e}^{S T} r_{e}^{G}\right\rangle=\rho r_{e}^{G},
$$

then $v_{1} \in \rho r_{e}^{S}$. Similarly $v_{2} \in \rho r_{e}^{T}$ and $v_{3} \in \rho r_{e}^{S T}$. If we replace $v_{1}=r_{e}^{S}$ in (7.2), we get $v_{2}=c_{g, e} r_{e}^{T}$ and $v_{3}=c_{h, e} r_{e}^{S T}$ for some $g, h \in G$ (check the multiplication table in the appendix). But $c_{g, e} r_{e}^{T}=t_{e}^{T}+\left(1_{e}+c_{g, e}\right) r_{e}^{T}$, where $\left(1_{e}+c_{g, e}\right) r_{e}^{T} \in \rho r_{T}^{G} t_{T}^{G}$, and similarly for $v_{3}$. This proves (7.3) which implies

$$
\text { ker } \partial_{1} \subseteq \rho\left\langle\left(r_{S}^{G} t_{S}^{G}, 0,0\right),\left(0, r_{T}^{G} t_{T}^{G}, 0\right),\left(0,0, r_{S T}^{G} t_{S T}^{G}\right),\left(r_{e}^{S}, r_{e}^{T}, r_{e}^{S T}\right)\right\rangle=\operatorname{Im} \partial_{2} .
$$

Lemma 7.5. Let $\partial_{0}^{\prime}: \rho 1_{e}^{2} \longrightarrow \rho 1_{e}, \partial_{1}^{\prime}: \widetilde{M} \longrightarrow \rho 1_{e}^{2}$ and $\partial_{2}^{\prime}: \widetilde{M} \oplus \rho 1_{G} \longrightarrow \widetilde{M}$ be the maps

and

$$
\partial_{0}^{\prime}=\left[\begin{array}{ll}
r_{e}^{S} t_{e}^{S} & r_{e}^{T} t_{e}^{T}
\end{array}\right], \quad \partial_{1}^{\prime}=\left[\begin{array}{ccc}
t_{e}^{S} & 0 & t_{e}^{S T} \\
0 & t_{e}^{T} & t_{e}^{S T}
\end{array}\right]
$$

$$
\partial_{2}^{\prime}=\left[\begin{array}{cccc}
t_{e}^{S} r_{e}^{S} & 0 & 0 & t_{S}^{G} \\
0 & t_{e}^{T} r_{e}^{T} & 0 & t_{T}^{G} \\
0 & 0 & t_{e}^{S T} r_{e}^{S T} & t_{S T}^{G}
\end{array}\right]
$$

Then

$$
\rho 1_{e} \stackrel{\partial_{0}^{\prime}}{\longleftarrow} \rho 1_{e}^{2} \stackrel{\partial_{1}^{\prime}}{\longleftarrow} \widetilde{M} \stackrel{\partial_{2}^{\prime}}{\longleftarrow} \widetilde{M} \oplus \rho 1_{G}
$$

is exact.

Proof. Apply the upside down functor $\mathcal{U}$ to Lemma 7.4

Proposition 7.6. There is a projective $\rho$-resolution for $\widetilde{F}$ of the form

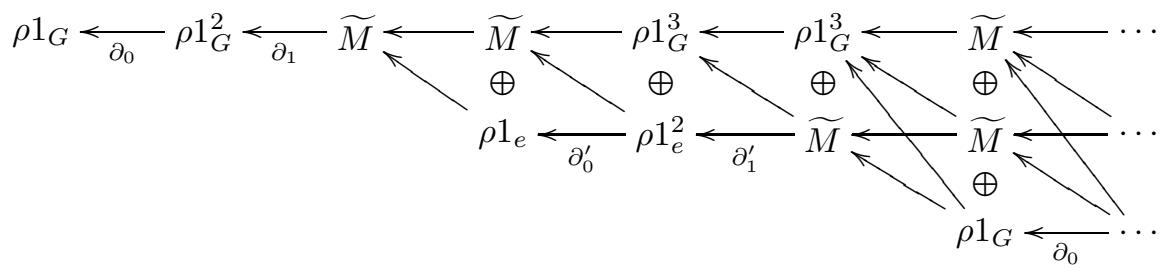

where each row becomes 4-periodic after the first two maps, and so do the correction maps between any two different rows. The maps $\partial_{0}, \partial_{1}, \partial_{0}^{\prime}$ and $\partial_{1}^{\prime}$ were defined in Lemmas 7.4 and 7.5 .

Proof. The exact sequence of Lemma 7.4 is the beginning of a resolution $P_{*}$ for $\widetilde{F}$. The idea is to apply the short exact sequence trick (Proposition 3.1) with $A=\operatorname{Im}\left(\left.\partial_{2}\right|_{\widetilde{M}}\right), B=\operatorname{Im} \partial_{2}$ and $C=B / A$. We only need projective resolutions for $A$ and $C$.

The map $\left.\partial_{2}\right|_{\widetilde{M}}: \widetilde{M} \longrightarrow \widetilde{M}$ is diagonal and each diagonal entry is $r_{H}^{G} t_{H}^{G}: \rho 1_{H} \longrightarrow$ $\rho 1_{H}(H=S, T$ or $S T)$, which occurs as a map in the exact sequence of Lemma 7.3 So a resolution for $A$ is obtained by taking the direct sum of the three long exact sequences of Lemma 7.3, and that is the first row of the resolution $P_{*}$.

By definition

$$
C=\operatorname{Im}\left(\left.\partial_{2}\right|_{\rho 1_{e}}\right) /\left(\operatorname{Im}\left(\left.\partial_{2}\right|_{\rho 1_{e}}\right) \cap \operatorname{Im}\left(\left.\partial_{2}\right|_{\widetilde{M}}\right)\right),
$$


where $\operatorname{Im}\left(\left.\partial_{2}\right|_{\rho 1_{e}}\right)=\rho\left(r_{e}^{S}, r_{e}^{T}, r_{e}^{S T}\right)$ and

$$
\operatorname{Im}\left(\left.\partial_{2}\right|_{\widetilde{M}}\right)=\rho\left(r_{S}^{G} t_{S}^{G}, 0,0\right) \oplus \rho\left(0, r_{T}^{G} t_{T}^{G}, 0\right) \oplus \rho\left(0,0, r_{S T}^{G} t_{S T}^{G}\right) .
$$

Using the multiplication table in the appendix, we get

$$
C=\mathbb{F}_{2}\left\langle\left(t_{e}^{H} r_{e}^{S}, t_{e}^{H} r_{e}^{T}, t_{e}^{H} r_{e}^{S T}\right): H \subseteq G\right\rangle .
$$

Now it is easy to see that $C$ is a $\rho$-module with $\operatorname{rank} 5,1_{H} C=\mathbb{F}_{2}$ for every subgroup $H, t_{K}^{H}$ acts by left multiplication and $r_{K}^{H}$ acts as zero if $H \neq K$, i.e. $C$ is isomorphic to $\mathcal{U}(\widetilde{F})=\mathbb{F}_{2}\left\langle t_{e}^{H}: H \subseteq G\right\rangle$. So $\mathcal{U}\left(P_{*}\right)$ is a projective resolution for $C$.

Next we will give a more detailed description of the maps in the resolution $P_{*}$. Everything follows from the way $P_{*}$ was constructed, namely the short exact sequence trick.

If we write the differential maps of $P_{*}$ as matrices as in Lemmas 7.4 and 7.5 , we get

$$
\partial_{3+i}=\left[\begin{array}{cc}
D_{3+i} & * \\
0 & \mathcal{U}\left(\partial_{i}\right)
\end{array}\right]
$$

for $i \geq 0$, where $D_{3+i}$ is a $3 \times 3$ block whose entries are the maps in the first row of the resolution for $F$, so the index $3+i$ should be taken mod 4, i.e. there are only four of these blocks and they appear periodicaly with period four. They are

$$
\begin{array}{lll}
D_{0}=\left[\begin{array}{lll}
t_{S}^{G} r_{S}^{G} & & \\
& t_{T}^{G} r_{T}^{G} & \\
& & t_{S T}^{G} r_{S T}^{G}
\end{array}\right], & D_{1}=\left[\begin{array}{lll}
t_{S}^{G} & & \\
& t_{T}^{G} & \\
& & t_{S T}^{G}
\end{array}\right], \\
D_{2}=\left[\begin{array}{lll}
r_{S}^{G} t_{S}^{G} & & \\
& r_{T}^{G} t_{T}^{G} & \\
& & r_{S T}^{G} t_{S T}^{G}
\end{array}\right], & D_{3}=\left[\begin{array}{lll}
r_{S}^{G} & & \\
& r_{T}^{G} & \\
& & r_{S T}^{G}
\end{array}\right] .
\end{array}
$$

These blocks correspond to the direct sum of the three long exact sequences of Lemma 7.3

If $i \geq 1$, the block $*$ has at least 3 columns, so we can write

$$
\partial_{3+i}=\left[\begin{array}{c|c}
D_{3+i} & A_{3+i} * \\
\hline 0 & \mathcal{U}\left(\partial_{i}\right)
\end{array}\right],
$$

where $A_{3+i}$ is a $3 \times 3$ block obtained by applying the short exact sequence trick once. Like the blocks in the diogonal, the $A_{3+i}$ are also 4-periodic. They are

$$
A_{0}=A_{1}=\left[\begin{array}{ccc}
0 & r_{T}^{G} & r_{S T}^{G} \\
r_{S}^{G} & 0 & r_{S T}^{G} \\
r_{S}^{G} & r_{T}^{G} & 0
\end{array}\right] \text { and } A_{2}=A_{3}=\left[\begin{array}{ccc}
0 & r_{e}^{S} & r_{e}^{S} \\
r_{e}^{T} & 0 & r_{e}^{T} \\
r_{e}^{S T} & r_{e}^{S T} & 0
\end{array}\right]
$$

The second application of the short exact sequence trick allows us to write the differential maps in the form

$$
\partial_{6+i}=\left[\begin{array}{ccc}
D_{6+i} & A_{6+i} & * \\
0 & \mathcal{U}\left(D_{6+i+1}\right) & * \\
0 & 0 & \partial_{i}
\end{array}\right]
$$

for $i \geq 0$.

If $i \geq 1$, then we can write

$$
\partial_{6+i}=\left[\begin{array}{c|c|cc}
D_{6+i} & A_{6+i} & B_{6+i} * \\
\hline 0 & \mathcal{U}\left(D_{6+i+1}\right) & \mathcal{U}\left(A_{6+i+1}\right) & * \\
\hline 0 & 0 & \partial_{i}
\end{array}\right],
$$


where

$$
\begin{gathered}
B_{0}=\left[\begin{array}{lll}
0 & & \\
& 0 & \\
& & t_{e}^{S T} r_{e}^{S T}
\end{array}\right], \quad B_{1}=\left[\begin{array}{ccc}
0 & r_{T}^{G} & r_{S T}^{G} \\
r_{S}^{G} & 0 & r_{S T}^{G} \\
r_{S}^{G} & r_{T}^{G} & 0
\end{array}\right], \\
B_{2}=\left[\begin{array}{ccc}
\lambda_{G, S T} & \lambda_{G, S T} & \lambda_{G, S T} \\
\lambda_{G, S} & \lambda_{G, S} & \lambda_{G, S} \\
\lambda_{G, T} & \lambda_{G, T} & 1_{G}
\end{array}\right], \\
B_{3}=\left[\begin{array}{ccc}
0 & t_{S}^{G} & t_{S}^{G}+t_{e}^{G} r_{e}^{S} \\
t_{T}^{G}+t_{e}^{G} r_{e}^{T} & 0 & t_{T}^{G} \\
t_{S T}^{G} & t_{S T}^{G}+t_{e}^{G} r_{e}^{S T} & t_{e}^{G} r_{e}^{S T}
\end{array}\right] .
\end{gathered}
$$

We can keep on breaking $\partial_{i}$ into $3 \times 3$ blocks, each of them occurring with period 4. But since $\partial_{i}$ is a $(i+1) \times(i+2)$ matrix, there are also blocks of size $1 \times 2,2 \times 3$, $3 \times 1$ and $3 \times 2$. The nonzero $1 \times 2$ blocks are either $\partial_{0}$ or $\partial_{0}^{\prime}$ and occur in the right lower corner of $\partial_{3 k}, k \geq 0$. The nonzero $2 \times 3$ blocks are either $\partial_{1}$ or $\partial_{1}^{\prime}$ and occur in the right lower corner of $\partial_{1+3 k}, k \geq 0$. The $3 \times 1$ blocks occur in the last column of $\partial_{2+3 k}, k \geq 0$. The $3 \times 2$ blocks occur in the last two columns of $\partial_{3 k}, k \geq 1$.

In Section 7.2 we will need the following differentials:

$$
\begin{gathered}
\partial_{2}=\left[\begin{array}{cc}
D_{2} & Z
\end{array}\right], \quad \partial_{3}=\left[\begin{array}{cc}
D_{3} & Y \\
0 & \partial_{0}^{\prime}
\end{array}\right], \quad \partial_{4}=\left[\begin{array}{cc}
D_{0} & A_{0} \\
0 & \partial_{1}^{\prime}
\end{array}\right], \\
\partial_{5}=\left[\begin{array}{ccc}
D_{1} & A_{1} & X \\
0 & \mathcal{U}\left(D_{2}\right) & \mathcal{U}(Z)
\end{array}\right], \quad \partial_{6}=\left[\begin{array}{ccc}
D_{2} & A_{2} & W \\
0 & \mathcal{U}\left(D_{3}\right) & \mathcal{U}(Y) \\
0 & 0 & \partial_{0}
\end{array}\right], \\
\partial_{7}=\left[\begin{array}{ccc}
D_{3} & A_{3} & B_{1} \\
0 & \mathcal{U}\left(D_{0}\right) & \mathcal{U}\left(A_{0}\right) \\
0 & 0 & \partial_{1}
\end{array}\right], \quad \partial_{8}=\left[\begin{array}{cccc}
D_{0} & A_{0} & B_{0} & V \\
0 & \mathcal{U}\left(D_{1}\right) & \mathcal{U}\left(A_{1}\right) & \mathcal{U}(X) \\
0 & 0 & D_{2} & Z
\end{array}\right]
\end{gathered}
$$

and

$$
\partial_{9}=\left[\begin{array}{cccc}
D_{1} & A_{1} & B_{1} & U \\
0 & \mathcal{U}\left(D_{2}\right) & \mathcal{U}\left(A_{2}\right) & \mathcal{U}(W) \\
0 & 0 & D_{3} & Y \\
0 & 0 & 0 & \partial_{0}^{\prime}
\end{array}\right]
$$

where

$$
\begin{array}{cc}
V=\left[\begin{array}{c}
r_{e}^{G} \\
r_{e}^{G} \\
r_{e}^{G}
\end{array}\right], \quad W=\left[\begin{array}{cc}
0 & t_{S}^{G} \\
t_{T}^{G}+t_{e}^{G} r_{e}^{T} & 0 \\
t_{S T}^{G} & t_{S T}^{G}+t_{e}^{G} r_{e}^{S T}
\end{array}\right], \\
X=\left[\begin{array}{c}
\lambda_{G, S T} \\
\lambda_{G, S} \\
\lambda_{G, T}
\end{array}\right], \quad Y=\left[\begin{array}{cc}
0 & r_{e}^{S} \\
r_{e}^{T} & 0 \\
r_{e}^{S T} & r_{e}^{S T}
\end{array}\right], \quad Z=\left[\begin{array}{c}
r_{e}^{S} \\
r_{e}^{T} \\
r_{e}^{S T}
\end{array}\right]
\end{array}
$$

and $U$ is 0 .

7.2. The algebra $\operatorname{Ext}_{R}(F, F)(G / G)$. Since

$$
\partial_{i}=\left[\begin{array}{cc}
* & * \\
0 & \partial_{i-6}
\end{array}\right]
$$

we can write $P_{i}=Q_{i} \oplus P_{i-6}$ for $i \geq 6$. Let $x: P_{*} \longrightarrow P[-6]_{*}$ be the chain map where $x_{i}$ is the projection $Q_{i} \oplus P_{i-6} \longrightarrow P_{i-6}, i \geq 6$. 
Lemma 7.7. Let $f: P_{*} \longrightarrow P[-n]_{*}$ be any chain map. Then $f \simeq 0$ iff $f x \simeq 0$.

Proof. Apply Lemma 3.2

Proposition 7.8. Let $\left(P_{*}, \partial_{*}\right)$ be the resolution in Proposition 7.6. There are chain maps $\alpha: P_{*} \longrightarrow P[-3]_{*}, \beta: P_{*} \longrightarrow P[-4]_{*}$ and $\gamma: P_{*} \longrightarrow P[-5]_{*}$, all not homotopic to zero, such that

(i) $\alpha, \beta$ and $\gamma$ commute with each other up to a chain homotopy,

(ii) $\alpha^{2} \simeq x$,

(iii) $\gamma^{2} \simeq 0$,

(iv) $\beta^{n} \not 0 \quad \forall n \geq 0$,

(v) $\alpha \beta^{n} \nsucceq 0 \quad \forall n \geq 0$,

(vi) $\gamma \beta^{n} \not 0 \quad \forall n \geq 0$,

(vii) $\gamma \alpha \beta^{n} \not 0$ and $\gamma \alpha \beta^{n} \not \beta^{n+2} \quad \forall n \geq 0$.

Proof. Let

$$
\begin{gathered}
\alpha_{3}=\left[\begin{array}{cccc}
r_{S}^{G} & r_{T}^{G} & r_{S T}^{G} & 0
\end{array}\right], \quad \beta_{4}=\left[\begin{array}{cccccc}
1_{G} & 1_{G} & 1_{G} & 0 & 0
\end{array}\right], \\
\gamma_{5}=\left[\begin{array}{lllllll}
0 & 0 & 0 & r_{S}^{G} & r_{T}^{G} & r_{S T}^{G}
\end{array}\right] .
\end{gathered}
$$

Since the image of $\alpha_{3} \partial_{3}$ is in the image of $\partial_{0}$, we can lift $\alpha_{3}: P_{3} \longrightarrow P_{0}$ to a chain map $\alpha: P_{*} \longrightarrow P[-3]_{*}$. Similarly, $\beta_{4}: P_{4} \longrightarrow P_{0}$ and $\gamma_{5}: P_{5} \longrightarrow P_{0}$ also define chain maps $\beta: P_{*} \longrightarrow P[-4]_{*}$ and $\gamma: P_{*} \longrightarrow P[-5]_{*}$.

Lemma 7.9. $\alpha, \beta$ and $\gamma$ are not chain homotopic to 0 .

Proof. By Lemma 3.2 $\alpha \simeq 0$ iff there is $\pi: \widetilde{M} \longrightarrow \widetilde{F}$ such that

$$
\phi \partial_{2}=\epsilon \alpha_{3}=\left[\begin{array}{llll}
r_{S}^{G} & r_{T}^{G} & r_{S T}^{G} & 0
\end{array}\right],
$$

where $\epsilon: \rho 1_{G} \longrightarrow \widetilde{F}$ is surjective. But all maps from $\widetilde{M}$ to $\widetilde{F}$ are of the form $\phi=\left[\begin{array}{lll}a_{1} & a_{2} & a_{3}\end{array}\right]$ with $a_{1}: \rho 1_{S} \longrightarrow \widetilde{F}$, so $a_{1}$ is either 0 or $r_{S}^{G}$. Similarly for $a_{2}$ and $a_{3}$. So $\phi \partial_{2}=\left[\begin{array}{llll}0 & 0 & 0 & *\end{array}\right] \neq \epsilon \alpha_{3}$ for all $\phi$.

The same argument works for $\beta$.

If $\gamma \simeq 0$, then there is $\phi: \rho 1_{G}^{3} \oplus \rho 1_{e}^{2} \longrightarrow \widetilde{F}$ such that

$$
\phi \partial_{4}=\epsilon \gamma_{5}=\left[\begin{array}{llllll}
0 & 0 & 0 & r_{S}^{G} & r_{T}^{G} & r_{S T}^{G}
\end{array}\right] .
$$

$\phi$ must be $\left[\begin{array}{lllll}a_{1} & a_{2} & a_{3} & a_{4} & a_{5}\end{array}\right]$ with $a_{i}=1_{G}$ or 0 for $i=1,2,3$, and $a_{i}=r_{e}^{G}$ or 0 for $i=4,5$. If $a_{4}=r_{e}^{G}$ and $a_{5}=0$, then

$$
\phi \partial_{4}=\left[\begin{array}{llllll}
0 & 0 & 0 & 0 & r_{T}^{G} & r_{S T}^{G}
\end{array}\right] .
$$

If $a_{4}=0$ and $a_{5}=r_{e}^{G}$, then $\phi \partial_{4}=\left[\begin{array}{llllll}0 & 0 & 0 & r_{S}^{G} & 0 & r_{S T}^{G}\end{array}\right]$. There are at most two nonzero entries in $\phi \partial_{4}$ and so $\phi \partial_{4} \neq \epsilon \gamma_{5}$ for all $\phi$. 
To prove parts (i), (ii) and (iii), we need the following liftings of the chain maps $\alpha, \beta$ and $\gamma$ :

$$
\begin{aligned}
& \alpha_{6}=\left[\begin{array}{lll|lll|l}
1_{S} & & & \multicolumn{3}{|c|}{t_{e}^{T} r_{e}^{T}} & \\
& 1_{T} & & & \\
& & 1_{S T} & & & & \\
\hline & & t_{e}^{S} & t_{e}^{T} & t_{e}^{S T} & t_{e}^{G}
\end{array}\right],
\end{aligned}
$$

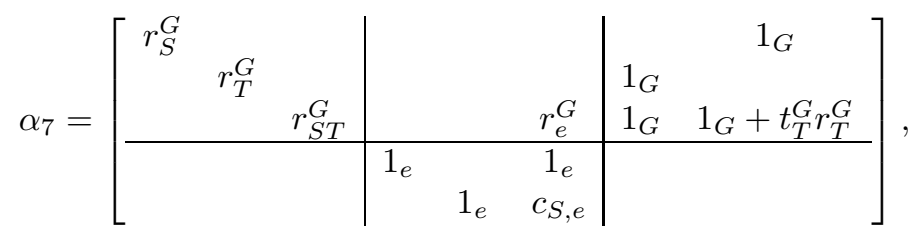

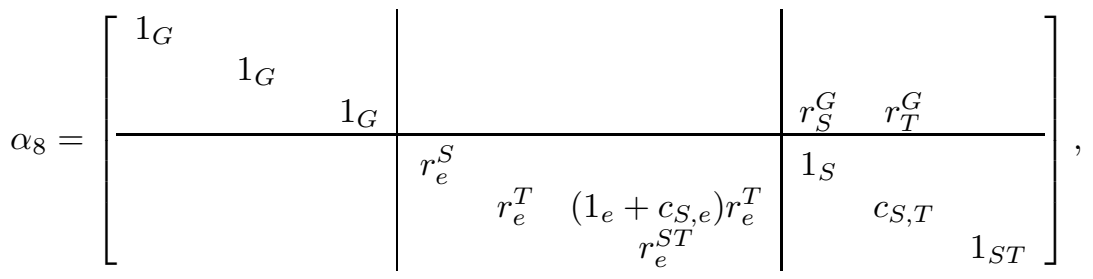

$$
\begin{aligned}
& \beta_{7}=\left[\begin{array}{lll|lll|l}
1_{S} & & & \multicolumn{2}{|c|}{r_{e}^{T}} & r_{e}^{T} & \\
& 1_{T} & & \\
& & 1_{S T} & & & \\
\hline & & 1_{e} & 1_{e} & 1_{e} & t_{e}^{G}
\end{array}\right],
\end{aligned}
$$

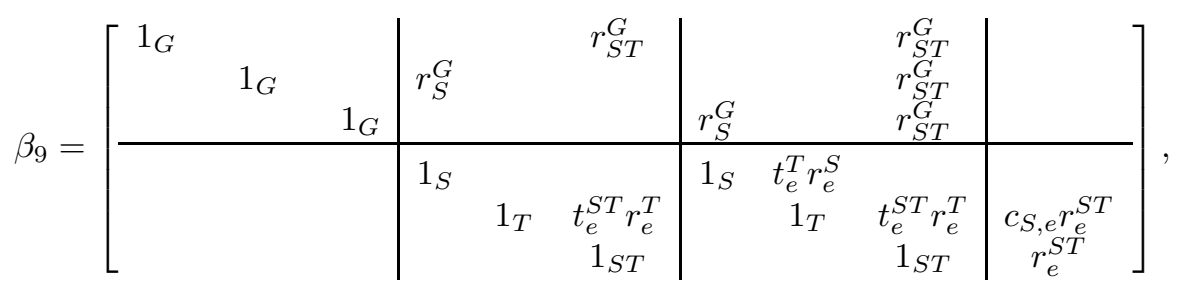

$$
\begin{aligned}
& \gamma_{8}=\left[\begin{array}{ccc|ccc|ccc}
0 & & & & r_{e}^{S} & 1_{S} & & \\
& 0 & & r_{e}^{T} & & & & 1_{T}+t_{e}^{T} r_{e}^{T} & \\
& & 0 & & r_{e}^{S T} & & & & 1_{S T} \\
\hline & & & & & t_{e}^{S} & t_{e}^{T} & t_{e}^{S T}
\end{array}\right], \\
& \gamma_{9}=\left[\begin{array}{ccc|ccc|ccc|c}
0 & & & & & r_{S T}^{G} & r_{S}^{G} & & & r_{e}^{G} \\
& 0 & & r_{S}^{G} & & & & r_{T}^{G} & & r_{e}^{G} \\
& & 0 & & r_{T}^{G} & & & r_{S T}^{G} & \\
\hline & & & & & & t_{e}^{T} c_{S, e} & t_{e}^{S T} & 1_{e}+c_{T, e}
\end{array}\right],
\end{aligned}
$$

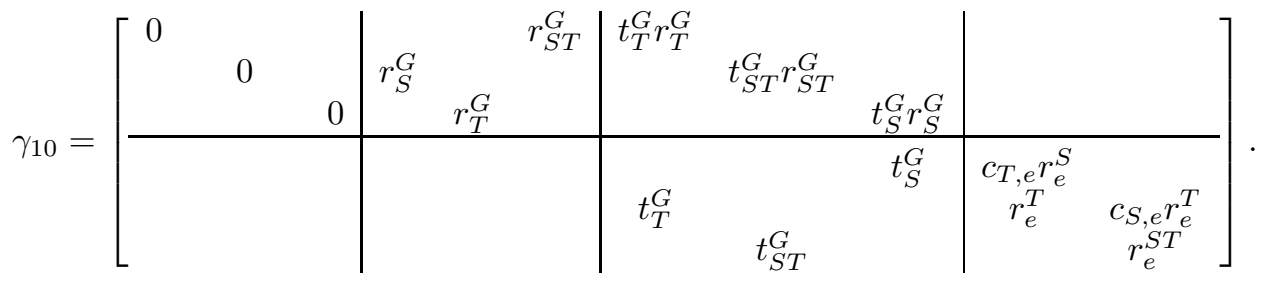


All of them are obtained by direct computation using the differential maps up to $\partial_{9}$ which were listed at the end of Section 7.1

Part (i): By Lemma [3.2, to see that $\alpha \beta \simeq \beta \alpha$, it is enough to find a $\phi$ such that $\phi \partial_{7}=\epsilon\left(\alpha_{3} \beta_{7}-\beta_{4} \alpha_{7}\right)$. In this case

$$
\phi=\left[\begin{array}{lllllll}
0 & 0 & 0 & r_{S}^{G} & r_{T}^{G} & r_{S T}^{G} & 0
\end{array}\right]
$$

does the job. Since $\epsilon\left(\alpha_{3} \gamma_{8}-\gamma_{5} \alpha_{8}\right)=0$ and $\epsilon\left(\beta_{4} \gamma_{9}-\gamma_{5} \beta_{9}\right)=0$, we can take $\phi=0$ to show that $\alpha \gamma \simeq \gamma \alpha$ and $\beta \gamma \simeq \gamma \beta$.

Part (ii): Let $\phi=\left[\begin{array}{llllll}1_{G} & 1_{G} & 1_{G} & 0 & 0 & 0\end{array}\right]$. Then

$$
\phi \partial_{5}=\epsilon\left(\alpha_{3} \alpha_{6}-x_{6}\right)=\left[\begin{array}{lllllll}
r_{S}^{G} & r_{T}^{G} & r_{S T}^{G} & 0 & 0 & 0 & 1_{G}
\end{array}\right],
$$

so $\alpha^{2} \simeq x$.

Part (iii): $\epsilon \gamma_{5} \gamma_{10}=0$, so $\gamma^{2} \simeq 0$.

To prove parts (iv) and (v) we need the following

Lemma 7.10. If $i \geq 7$, then

$$
\beta_{i}=\left[\begin{array}{lll|l}
1 & & & \\
& 1 & & * \\
& & 1 & \\
\hline & 0 & & *
\end{array}\right] .
$$

Proof. Suppose it is true for $\beta_{i}$. Recall that

$$
\partial_{i}=\left[\begin{array}{cc}
D_{i} & * \\
0 & *
\end{array}\right] \quad \text { and } \quad \partial_{i-4}=\left[\begin{array}{cc}
D_{i-4} & * \\
0 & *
\end{array}\right]
$$

with $D_{i-4}=D_{i}$. $\beta_{i+1}$ is a map that satisfies $\beta_{i} \partial_{i}=\partial_{i-4} \beta_{i+1}$, so it can be of the form (7.4).

From Lemma 7.10, we have

$$
\alpha_{3} \beta_{7} \cdots \beta_{3+4 n}=\left[\begin{array}{llll}
r_{S}^{G} & r_{T}^{G} & r_{S T}^{G} & *
\end{array}\right]
$$

and

$$
\beta_{4} \beta_{8} \cdots \beta_{4+4 n}=\left[\begin{array}{llll}
1_{G} & 1_{G} & 1_{G} & *
\end{array}\right] .
$$

Now the proof that shows $\alpha \neq 0$ and $\beta \neq 0$ also works in this case, and so we get $\alpha \beta^{n} \nsucceq 0$ and $\beta^{n} \nsucceq 0$.

Lemma 7.11. If $i \geq 10$, then

$$
\beta_{i}=\left[\begin{array}{lll|lll|l}
1 & & & & & \\
& 1 & & & * & & * \\
& & 1 & & & & \\
\hline & & & 1 & & & \\
& & & & 1 & & * \\
& & & & & 1 & \\
\hline & & & & & *
\end{array}\right] .
$$


Proof. $\beta_{10}$ can be computed directly from $\beta_{9}$. One possible lifting is

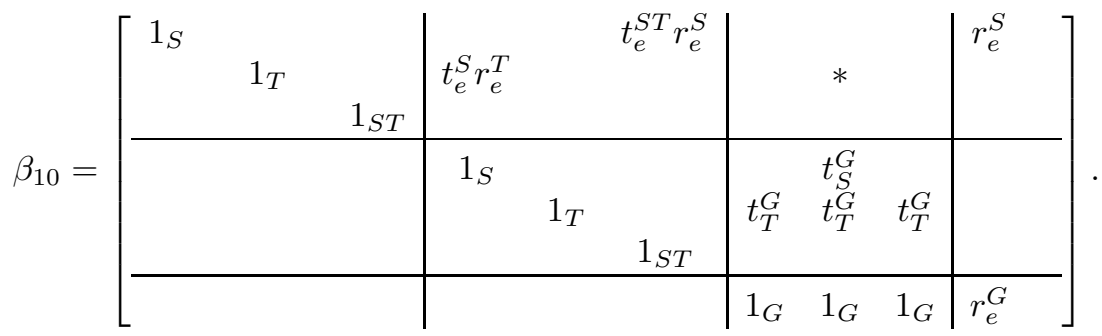

The result now follows by induction as in Lemma 7.10 but using

$$
\partial_{i}=\left[\begin{array}{ccc}
D_{i} & A_{i} & * \\
0 & \mathcal{U}\left(D_{i+1}\right) & * \\
0 & 0 & *
\end{array}\right] .
$$

Part (vi): From Lemma 7.11

$$
\gamma_{5} \beta_{9} \cdots \beta_{5+4 n}=\left[\begin{array}{lllllll}
0 & 0 & 0 & r_{S}^{G} & r_{T}^{G} & r_{S T}^{G} & *
\end{array}\right]
$$

so, as in parts (iv) and (v), the proof that $\gamma \neq 0$ also shows that $\gamma \beta^{n} \nsucceq 0$.

Part (vii): If $\gamma \alpha \beta^{n} \simeq 0$, then there is $\phi: \widetilde{M} \oplus \rho 1_{e}^{3} \oplus \cdots \longrightarrow \widetilde{F}$ such that $\phi \partial_{7+4 n}=\epsilon \gamma_{5} \alpha_{8}$. Since

$$
\epsilon \gamma_{5} \alpha_{8} \beta_{12} \beta_{16} \cdots \beta_{8+4 n}=\left[\begin{array}{lllllll}
0 & 0 & 0 & r_{e}^{G} & r_{e}^{G} & r_{e}^{G} & *
\end{array}\right],
$$

it is enough to consider $\phi$ of the form $\left[\begin{array}{lllllll}0 & 0 & 0 & a_{1} & a_{2} & a_{3} & *\end{array}\right]$ with $a_{i}: \rho 1_{e} \longrightarrow$ $\widetilde{F}$. So $a_{i}$ is either 0 or $r_{e}^{G}$. But

$$
\phi \partial_{7+4 n}=\left[\begin{array}{lllllll}
0 & 0 & 0 & 0 & 0 & 0 & *
\end{array}\right] \neq \epsilon \gamma_{5} \alpha_{8} \beta_{12} \beta_{16} \cdots \beta_{8+4 n}
$$

for all possible $\phi$ because $\left(1_{e}+c_{x, e}\right) r_{e}^{G}=0$ for all $x \in G$. This shows that $\gamma \alpha \beta^{n} \nsucceq 0$.

To prove that $\gamma \alpha \beta^{n} \not \beta^{n+2}$, do the same as in part (v). It works because

$$
\epsilon\left(\gamma_{5} \alpha_{8} \beta_{12} \cdots \beta_{8+4 n}-\beta_{4} \beta_{8} \cdots \beta_{8+4 n}\right)=\left[\begin{array}{llll}
1_{G} & 1_{G} & 1_{G} & *
\end{array}\right] .
$$

Corollary 7.12. The algebra $\operatorname{Ext}_{\rho}(\widetilde{F}, \widetilde{F})$ contains a subalgebra isomorphic to

$$
\mathbb{F}_{2}\left[y_{3}, x_{4}, y_{5}\right] /\left(y_{5}^{2}=0\right),
$$

where $\operatorname{deg}\left(y_{i}\right)=i$ and $\operatorname{deg}\left(x_{4}\right)=4$.

Let $Q_{i}=\operatorname{Hom}_{\rho}\left(P_{i}, \widetilde{F}\right), \delta_{i}=\operatorname{Hom}_{\rho}\left(\partial_{i}, \widetilde{F}\right)$ and $\delta_{i}^{\prime}=\operatorname{Hom}_{\rho}\left(\mathcal{U}\left(\partial_{i}\right), \widetilde{F}\right)$. By Proposition 5.3, $Q_{i}=\mathbb{F}_{2}^{i+1}$. Let $Q=\bigoplus Q_{i}$. Keeping track of the blocks of $\delta_{i}$, which are induced by the blocks of $\partial_{i}$ discussed at the end of Section 7.1 after applying the functor $\operatorname{Hom}_{\rho}(\cdot, \widetilde{F})$ to the resolution of Proposition [7.6, we get the following picture where each arrow is one block:

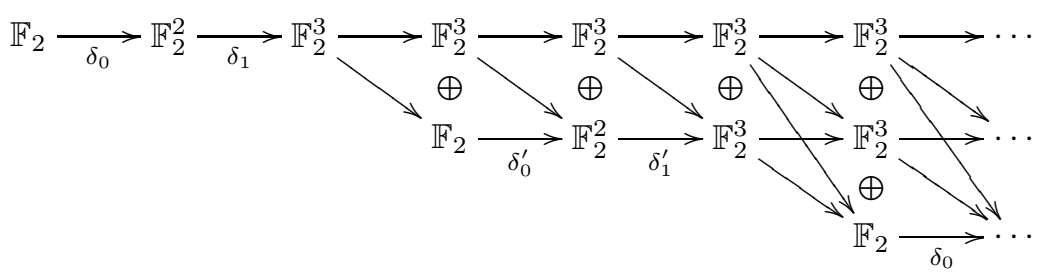

This block decomposition defines a decreasing filtration $F^{0} Q \supset F^{1} Q \supset \cdots \supset$ $F^{p} Q \supset \cdots$ of $Q$, where $F^{0} Q=Q, F^{1} Q$ is $Q$ without the first row of blocks, i.e. 
the first row of (7.5), in general $F^{p} Q$ is $Q$ without the first $p$ rows of blocks. There is a spectral sequence associated to the filtration with $E_{1}^{p, q}=H^{p+q}\left(F^{p} Q / F^{p+1} Q\right)$ converging to $H(Q)=\operatorname{Ext}_{\rho}(\widetilde{F}, \widetilde{F})$ (Theorem 2.6 in $[6]$ ). The differentials $d_{1}$ of the spectral sequence are $\operatorname{Hom}_{\rho}\left(A_{i}, \widetilde{F}\right)\left(A_{i}\right.$ are the blocks of $\partial_{i}$ above the blocks in the diagonal), the $d_{2}$ are $\operatorname{Hom}_{\rho}\left(B_{i}, \widetilde{F}\right)\left(B_{i}\right.$ are the blocks above the $\left.A_{i}\right)$, etc.

Lemma 7.13. If $p$ is even, then

$$
H^{i}\left(F^{p} Q / F^{p+1} Q\right)=\left\{\begin{array}{cl}
0 & i<3 p \\
\mathbb{F}_{2} & i=3 p, \\
0 & i=3 p+1, \\
\mathbb{F}_{2} & i=3 p+2, \\
\mathbb{F}_{2}^{3} \quad & i=3 p+3+4 k \text { or } 3 p+4+4 k, k \geq 0, \\
0 & i=3 p+5+4 k \text { or } 3 p+6+4 k, k \geq 0 .
\end{array}\right.
$$

If $p$ is odd, then

$$
H^{i}\left(F^{p} Q / F^{p+1} Q\right)=\left\{\begin{array}{cl}
0 & i<3 p \\
\mathbb{F}_{2} & i=3 p, \\
\mathbb{F}_{2}^{2} & i=3 p+1 \\
\mathbb{F}_{2}^{3} & i=3 p+2+4 k \text { or } 3 p+5+4 k, k \geq 0, \\
0 & i=3 p+3+4 k \text { or } 3 p+4+4 k, k \geq 0 .
\end{array}\right.
$$

Proof. The quotient $F^{p} Q / F^{p+1} Q$ is the $p$-th row of blocks of (7.5), which is the image of the $p$-th row of $P_{*}$ under the functor $\operatorname{Hom}_{\rho}(\cdot, \widetilde{F})$. So, if $p$ is even, then $H^{i}\left(F^{p} Q / F^{p+1} Q\right)$ is the cohomology of the complex

$$
\mathbb{F}_{2} \stackrel{\delta_{0}}{\longrightarrow} \mathbb{F}_{2}^{2} \stackrel{\delta_{1}}{\longrightarrow} \mathbb{F}_{2}^{3} \stackrel{d_{0}}{\longrightarrow} \mathbb{F}_{2}^{3} \stackrel{d_{1}}{\longrightarrow} \mathbb{F}_{2}^{3} \stackrel{d_{2}}{\longrightarrow} \mathbb{F}_{2}^{3} \stackrel{d_{3}}{\longrightarrow} \mathbb{F}_{2}^{3} \stackrel{d_{0}}{\longrightarrow} \cdots
$$

shifted by $3 p$, where $d_{i}=\operatorname{Hom}_{\rho}\left(D_{i}\right)$. Also, if $p$ is odd, then $H^{i}\left(F^{p} Q / F^{p+1} Q\right)$ is the cohomology of

$$
\mathbb{F}_{2} \stackrel{\delta_{0}^{\prime}}{\longrightarrow} \mathbb{F}_{2}^{2} \stackrel{\delta_{1}^{\prime}}{\longrightarrow} \mathbb{F}_{2}^{3} \stackrel{d_{0}^{\prime}}{\longrightarrow} \mathbb{F}_{2}^{3} \stackrel{d_{1}^{\prime}}{\longrightarrow} \mathbb{F}_{2}^{3} \stackrel{d_{2}^{\prime}}{\longrightarrow} \mathbb{F}_{2}^{3} \stackrel{d_{3}^{\prime}}{\longrightarrow} \mathbb{F}_{2}^{3} \stackrel{d_{0}^{\prime}}{\longrightarrow} \cdots
$$

shifted by $3 p$, where $d_{i}^{\prime}=\operatorname{Hom}_{\rho}\left(\mathcal{U}\left(D_{i}\right)\right)$.

Corollary 7.14. The $E_{1}$-term of the spectral sequence is

$$
\begin{aligned}
& E_{1}^{0, q}=\left\{\begin{array}{cl}
\mathbb{F}_{2} & \text { if } q=0, \\
0 & \text { if } q=1, \\
\mathbb{F}_{2} & \text { if } q=2, \\
\mathbb{F}_{2}^{3} & \text { if } q=3+4 k \text { or } 4+4 k, k \geq 0, \\
0 & \text { if } q=5+4 k \text { or } 6+4 k, k \geq 0,
\end{array}\right. \\
& E_{1}^{1, q}=\left\{\begin{array}{cl}
0 & \text { if } q=0,1, \\
\mathbb{F}_{2} & \text { if } q=2, \\
\mathbb{F}_{2}^{2} & \text { if } q=3, \\
\mathbb{F}_{2}^{3} & \text { if } q=4 k \text { or } 3+4 k, k \geq 1, \\
0 & \text { if } q=1+4 k \text { or } 2+4 k, k \geq 1,
\end{array}\right.
\end{aligned}
$$

and $E_{1}^{p+2, q+4}=E_{1}^{p, q}$ for all $p, q \geq 0$. 


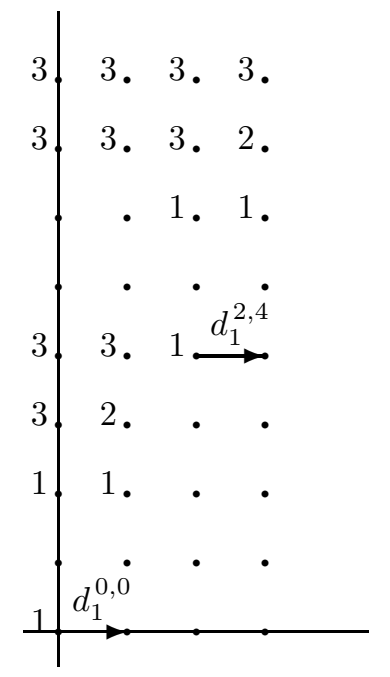

$E_{1}$-term:

The numbers are the ranks of each $E_{1}^{p, q}$. No number means the rank is zero.

Proposition 7.15. There is a map $x: E_{r} \longrightarrow E_{r}$ of graded modules such that $x\left(E_{r}^{p, q}\right) \subseteq E_{r}^{p+2, q+4}$.

Proof. Since the chain map $x: P_{*} \longrightarrow P[-6]_{*}$ preserves the filtration of $P_{*}, x$ acts on the $E_{r}$-terms of the spectral sequence increasing the degree by $6 . x$ represents a class in $\operatorname{Ext}_{\rho}(\widetilde{F}, \widetilde{F})$, so $x: E_{r} \longrightarrow E_{r}$ is not the zero map, and it must send $1 \in E_{r}^{0,0}$ to a class in $E_{r}$ of degree 6 . The only nonzero class of degree 6 in $E_{1}$ lies in $E_{1}^{2,4}$, so $x: E_{r}^{p, q} \longrightarrow E_{r}^{p+2, q+4}$, for all $p, q \geq 0$.

Lemma 7.16. The $E_{2}$-term of the spectral sequence is

$$
\begin{aligned}
& E_{2}^{0, q}=\left\{\begin{array}{cl}
\mathbb{F}_{2} & q=4 k \text { or } 3+4 k, k \geq 0, \\
0 & \text { otherwise },
\end{array}\right. \\
& E_{2}^{1, q}=\left\{\begin{array}{cl}
\mathbb{F}_{2} & q=4 k \text { or } 3+4 k, k \geq 1, \\
0 & \text { otherwise },
\end{array}\right.
\end{aligned}
$$

and $E_{2}^{p+2, q+4}=E_{2}^{p, q}$ for all $p, q \geq 0$.

Proof. From Lemma 5.3 we have

$$
\operatorname{Hom}(Z)=\left[\begin{array}{lll}
1 & 1 & 1
\end{array}\right], \quad \operatorname{Hom}(Y)=\left[\begin{array}{lll}
0 & 1 & 1 \\
1 & 0 & 1
\end{array}\right], \quad \operatorname{Hom}\left(A_{i}\right)=\left[\begin{array}{lll}
0 & 1 & 1 \\
1 & 0 & 1 \\
1 & 1 & 0
\end{array}\right]
$$

and

$$
\operatorname{Hom}(\mathcal{U}(Z))=0, \quad \operatorname{Hom}(\mathcal{U}(Y))=0, \quad \operatorname{Hom}\left(\mathcal{U}\left(A_{i}\right)\right)=0 .
$$

The differentials $d_{1}^{1, q}$ are induced by the maps $\operatorname{Hom}(\mathcal{U}(Z))$, $\operatorname{Hom}(\mathcal{U}(Y))$ and $\operatorname{Hom}\left(\mathcal{U}\left(A_{i}\right)\right)$, so they are zero.

The differential $d_{1}^{0,2}: E_{1}^{0,2} \longrightarrow E_{1}^{1,2}$ is the restriction of $\operatorname{Hom}(Z)$ to the quotient $E_{1}^{0,2}=\operatorname{ker} d_{0} / \operatorname{Im} \delta_{1}$ which is generated by the class $(1,1,1)$ (recall that $E_{1}^{0, q}$ is the homology of the complex (7.6) $)$. Since $\operatorname{Hom}(Z)(1,1,1)=1$, then $d_{1}^{0,2}=1$. 
From (7.6) and (7.7), we see that $E_{1}^{p, q}$ is a quotient by 0 if $q=3+4 k$ or $4+4 k$, $k \geq 0$ and $p=0,1$, so $d_{1}^{0,3}=\operatorname{Hom}(Y)$ and $d_{1}^{0, q}=\operatorname{Hom}\left(A_{q}\right)$, for $q=7+4 k, 4+4 k$, $k \geq 0$.

All other $d_{1}^{0, q}: E_{1}^{0, q} \longrightarrow E_{1}^{1, q}$ are zero because $E_{1}^{0, q}=0$ or $E_{1}^{1, q}=0$.

Now it is easy to compute $E_{2}^{p, q}=\operatorname{ker} d_{1}^{p, q} / \operatorname{Im} d_{1}^{p-1, q}$ for $p=0,1\left(\right.$ set $d_{1}^{p, q}=0$ if $p<0)$.

Finally, $E_{2}^{p+2, q+4}=E_{2}^{p, q}$ because the same is true for the $E_{1}$-term, $d^{1, q}=0$, and the maps $d_{1}^{p+2, q+4}$ and $d_{1}^{p, q}$ are induced by the same differential map of the resolution $P_{*}$, hence they are equal.

Theorem 7.17. $\operatorname{Ext}_{\rho}(\widetilde{F}, \widetilde{F}) \simeq \mathbb{F}_{2}\left[y_{3}, x_{4}, y_{5}\right] /\left(y_{5}^{2}=0\right)$.

Proof. Corollary 7.12 says that $\operatorname{Ext}_{\rho}(\widetilde{F}, \widetilde{F})$ is at least the graded algebra

$$
B=\mathbb{F}_{2}\left[y_{3}, x_{4}, y_{5}\right] /\left(y_{5}^{2}=0\right) .
$$

To show that $\operatorname{Ext}_{\rho}(\widetilde{F}, \widetilde{F})$ is at most $B$, it is enough to check that the rank of $\operatorname{Ext}_{\rho}^{i}(\widetilde{F}, \widetilde{F})$ is less than or equal to the rank of $B_{i}$ for all degrees $i$.

Since the spectral sequence converges to $\operatorname{Ext}_{\rho}(\widetilde{F}, \widetilde{F})$, we have

$$
r k\left(\operatorname{Ext}_{\rho}^{i}(\widetilde{F}, \widetilde{F})\right) \leq r k\left(\bigoplus_{p+q=i} E_{2}^{p, q}\right) .
$$

Let $f: B \longrightarrow \bigoplus_{p, q \geq 0} E_{2}^{p, q}$ be defined by $f\left(x_{4}^{n}\right)=1 \in E_{2}^{0,4 n}, f\left(y_{3} x_{4}^{n}\right)=1 \in$ $E_{2}^{0,3+4 n}, f\left(y_{5} x_{4}^{n}\right)=1 \in E_{2}^{1,4(n+1)}, f\left(y_{5} y_{3} x_{4}^{n}\right)=1 \in E_{2}^{1,3+4(n+1)}, f(x b)=1 \epsilon$ $E_{2}^{p+2, q+4}$ if $f(b)=1 \in E_{2}^{p, q}$, for all $n \geq 0$, i.e. $f$ is a map of graded modules that sends $x_{4}^{n}$ and $y_{3} x_{4}^{n}$ into $E_{2}^{0, q}$, sends the previous elements multiplied by $y_{5}$ into $E_{2}^{1, q}$, and commutes with the action of $x$. Here is the picture of the image of $f$ :

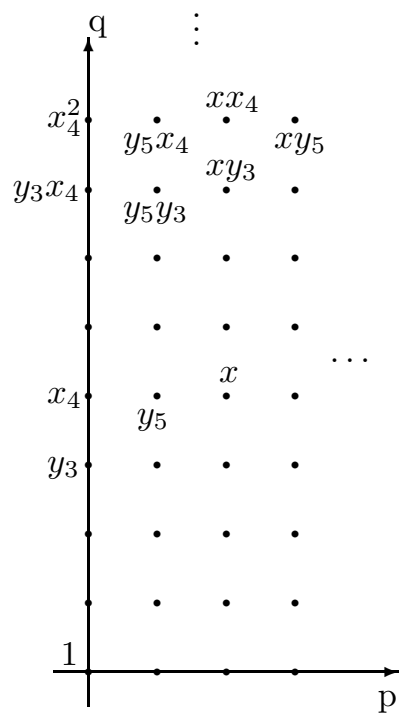

Lemma 7.16 implies that $f$ is an isomorphism, so $r k\left(\bigoplus_{p+q=i} E_{2}^{p, q}\right)=r k\left(B_{i}\right)$. 
7.3. The Green functor $\operatorname{Ext}_{R}(F, F)$. Proposition 6.4, Theorem 7.17 and Corollaries 4.9 and 4.10 give the value of $\operatorname{Ext}_{R}(F, F)$ on the objects of $\mathcal{B}$ :

$$
\begin{aligned}
\operatorname{Ext}_{R}(F, F)(G / G) & =\mathbb{F}_{2}\left[y_{3}, x_{4}\right] \otimes E\left(y_{5}\right), \\
\operatorname{Ext}_{R}(F, F)(G / H) & =\mathbb{F}_{2}\left[x_{4}\right] \otimes E\left(y_{3}\right) \text { for } H \text { of index 2, } \\
\operatorname{Ext}_{R}(F, F)(G / e) & =0 .
\end{aligned}
$$

The only thing left is to find the morphisms $r_{H}^{G}$ and $t_{H}^{G}$.

Proposition 7.18. The restriction map $r_{H}^{G}$ is the ring map that sends $y_{3}, x_{4}$ to the elements in $\operatorname{Ext}_{R}(F, F)(G / H)$ with the same name and sends $y_{5}$ to zero. The induction map $t_{H}^{G}$ is zero.

Proof. The spectral sequence used to compute $\operatorname{Ext}_{R}(F, F)(G / G)$ comes from a filtration of the complex (7.5) which was obtained from the resolution of Proposition 7.6 by applying $\operatorname{Hom}_{\rho}(\cdot, \widetilde{F})$. This was all done in the category of modules over $\rho$. If we translate the resolution in Proposition[7.6 into the language of Mackey functors, we obtain a projective resolution for the module $F$ over the Green functor $R$. Now apply the functor $\mathcal{H}_{R}(\cdot, F)$ to this resolution to get a complex of $R$-modules which looks like (77.5) with $\mathbb{F}_{2}$ replaced by $F$. This complex can be filtered in the same way (7.5) was and we get a spectral sequence of $R$-modules converging to the graded $R$-module $\operatorname{Ext}_{R}(F, F)$. The "old" spectral sequence is the value of this one at the $G$-set $G / G$, so we know that the "new" spectral sequence collapses at the $E_{2}$-term. A direct computation of the $E_{2}$-term gives $\operatorname{Ext}_{R}(F, F)$ as an $R$-module, including the above descriptions of $r_{H}^{G}$ and $t_{H}^{G}$. This is made easier since we already know that $\operatorname{Ext}_{R}(F, F)$ is a Green functor, which implies that $r_{H}^{G}$ is a ring map, so we only need to know its value on the generators $y_{3}, x_{4}$ and $y_{5}$. Surjectivity of $r_{H}^{G}$ implies that $t_{H}^{G}$ is zero.

\section{Appendix A. Multiplication tables}

Let $G=\mathbb{Z} / 2 \times \mathbb{Z} / 2$. Let $H, K$ and $L$ be subgroups of order 2. Let $x \in G / H$, $x \notin H, y \in G / K, y \notin K, z \in G / L, z \notin L$. Tables $1-3$ contain the nonzero products of the generators of the Mackey algebra $\mu=\mu_{\mathbb{F}_{2}}(G)$.

\section{TABLE 1.}

\begin{tabular}{c|ccc} 
& $c_{g, e}$ & $c_{g, e} r_{e}^{H}$ & $r_{e}^{G}$ \\
\hline$c_{h, e}$ & $c_{h g, e}$ & $c_{h g, e} r_{e}^{H}$ & $r_{e}^{G}$ \\
$t_{e}^{K} c_{h, e}$ & $t_{e}^{K} c_{h g, e}$ & $\left\{\begin{array}{c}t_{e}^{K} r_{e}^{H}, \text { if } H \neq K \\
t_{e}^{H} c_{h g, e} r_{e}^{H}, \text { if } H=K\end{array}\right.$ & $t_{e}^{K} r_{e}^{G}$ \\
$t_{e}^{G}$ & $t_{e}^{G}$ & $t_{e}^{G} r_{e}^{H}$ & $t_{e}^{G} r_{e}^{G}$
\end{tabular}


TABLE 2 .

\begin{tabular}{|c|c|c|c|c|c|c|}
\hline & $t_{e}^{H} c_{g, e}$ & $c_{g, H}$ & $t_{e}^{H} c_{g, e} r_{e}^{H}$ & $\begin{array}{c}t_{e}^{H} r_{e}^{L} \\
L \neq H\end{array}$ & $r_{H}^{G}$ & $t_{e}^{H} r_{e}^{G}$ \\
\hline$c_{h, e} r_{e}^{H}$ & $\sum_{h \in H} c_{h a g, e}$ & $c_{h g, e} r_{e}^{H}$ & 0 & $\left(1_{e}+c_{z, e}\right) r_{e}^{L}$ & $r_{e}^{G}$ & 0 \\
\hline$c_{h, H}$ & $\begin{array}{l}a \in H \\
t_{e}^{H} \\
c_{h g, e}\end{array}$ & $c_{h g, H}$ & $t_{e}^{H} c_{h g, e} r_{e}^{H}$ & $t_{e}^{H} r_{e}^{L}$ & $r_{H}^{G}$ & $t_{e}^{H} r_{e}^{G}$ \\
\hline$t_{e}^{H} c_{h, e} r_{e}^{H}$ & 0 & $t_{e}^{H} c_{h g, e} r_{e}^{H}$ & 0 & 0 & $t_{e}^{H} r_{e}^{G}$ & 0 \\
\hline $\begin{array}{c}t_{e}^{K} r_{e}^{H} \\
K \neq H\end{array}$ & $t_{e}^{K}\left(1_{e}+c_{y, e}\right)$ & $t_{e}^{K} r_{e}^{H}$ & 0 & $\left\{\begin{array}{c}t_{e}^{K}\left(1_{e}+c_{y, e}\right) r_{e}^{K} \\
\text { if } K=L\end{array}\right.$ & $t_{e}^{K} r_{e}^{G}$ & 0 \\
\hline$t_{H}^{G}$ & $t_{e}^{G}$ & $t_{H}^{G}$ & $t_{e}^{G} r_{e}^{H}$ & U, if $\Lambda \neq L$ & $t_{H}^{G} r_{H}^{G}$ & $t_{e}^{G} r_{e}^{G}$ \\
\hline$t_{e}^{G} r_{e}^{H}$ & 0 & $t_{e}^{G} r_{e}^{H}$ & 0 & 0 & $t_{e}^{G} r_{e}^{G}$ & 0 \\
\hline
\end{tabular}

TABLE 3 .

\begin{tabular}{c|cccccc} 
& $t_{e}^{G}$ & $t_{H}^{G}$ & $t_{e}^{G} r_{e}^{H}$ & $1_{G}$ & $t_{H}^{G} r_{H}^{G}$ & $t_{e}^{G} r_{e}^{G}$ \\
\hline$r_{e}^{G}$ & $\sum_{g \in G} c_{g, e}$ & $\left(1_{e}+c_{x, e}\right) r_{e}^{H}$ & 0 & $r_{e}^{G}$ & 0 & 0 \\
$r_{H}^{G}$ & $t_{e}^{H}\left(1_{e}+c_{x, e}\right)$ & $1_{H}+c_{x, H}$ & $t_{e}^{H}\left(1_{e}+c_{x, e}\right) r_{e}^{H}$ & $r_{H}^{G}$ & 0 & 0 \\
$t_{e}^{H} r_{e}^{G}$ & 0 & $t_{e}^{H}\left(1_{e}+c_{x, e}\right) r_{e}^{H}$ & 0 & $t_{H}^{G} r_{e}^{G}$ & 0 & 0 \\
$r_{K}^{G}$ & $t_{e}^{K}\left(1_{e}+c_{y, e}\right)$ & $t_{e}^{K} r_{e}^{H}$ & 0 & $r_{K}^{G}$ & $t_{e}^{K} r_{e}^{G}$ & 0 \\
$K \neq H$ & & & & \\
$t_{e}^{K} r_{e}^{G}$ & 0 & 0 & 0 & $t_{e}^{K} r_{e}^{G}$ & 0 & 0 \\
$K_{F} \neq H$ & $t_{e}^{G}$ & $t_{H}^{G}$ & $t_{e}^{G} r_{e}^{H}$ & $1_{G}$ & $t_{H}^{G} r_{H}^{G}$ & $t_{e}^{G} r_{e}^{G}$ \\
$1_{G}^{G}$ & 0 & 0 & 0 & $t_{H}^{G} r_{H}^{G}$ & 0 & 0 \\
$t_{H}^{G} r_{H}^{G}$ & 0 & $t_{e}^{G} r_{e}^{H}$ & 0 & $t_{K}^{G} r_{K}^{G}$ & $t_{e}^{G} r_{e}^{G}$ & 0 \\
$t_{K}^{G} r_{K}^{G}$ & 0 & 0 & 0 & $t_{e}^{G} r_{e}^{G}$ & 0 & 0 \\
$K_{F}^{G} H$ & 0 & & & & &
\end{tabular}

\section{REFERENCES}

1. Serge Bouc. Green functors and $G$-sets. Springer-Verlag, Berlin, 1997. MR 99c:20010

2. Andreas W. M. Dress. Contributions to the theory of induced representations. In Algebraic K-theory, II: "Classical" algebraic K-theory and connections with arithmetic (Proc. Conf., Battelle Memorial Inst., Seattle, Wash., 1972), pages 183-240. Lecture Notes in Math., Vol. 342. Springer, Berlin, 1973. MR 52:5787

3. L. Gaunce Lewis, Jr. The box product of Mackey functors. Unpublished.

4. L. Gaunce Lewis, Jr. The theory of Green functors. Unpublished notes, 1981.

5. Saunders Mac Lane. Categories for the working mathematician. Springer-Verlag, New York, second edition, 1998. MR 2001j:18001

6. John McCleary. A user's guide to spectral sequences. Cambridge University Press, Cambridge, second edition, 2001. MR 2002c:55027

7. Jacques Thévenaz and Peter Webb. The structure of Mackey functors. Trans. Amer. Math. Soc., 347(6):1865-1961, 1995. MR 95i:20018 
8. Jacques Thévenaz and Peter Webb. Simple Mackey functors. In Proceedings of the Second International Group Theory Conference (Bressanone, 1989), number 23, pages 299-319, 1990. MR 91g:20011

9. Charles A. Weibel. An introduction to homological algebra. Cambridge University Press, Cambridge, 1994. MR 95f:18001

Departamento de Matemática, Instituto Superior Técnico, Av. Rovisco Pais, 1049-001 Lisboa, Portugal

E-mail address: jventura@math.ist.utl.pt 